Type of the Paper (Article)

\title{
Flattening the curve of flexible space robotics
}

\author{
Timothy Sands ${ }^{1, *}$
}

1,* Sibley School of Mechanical and Aerospace Engineering, Cornell University, USA; tas297@cornell.edu

\begin{abstract}
Infrastructure monitoring, inspection, repair, and replacement in space is crucial for continued usage and safety, yet it is expensive, time-consuming, and technical very challenging. New robotics technologies and artificial intelligence algorithms are potentially novel approaches that may alleviate such demanding operations using existing or novel sensing technologies. Space structures must necessarily be very light weight due to high costs of placing robots in space. Several methods are proposed and compared to control highly flexible space robotics, where a key challenge is the presence of flexible resonant modes at frequencies so low as to reside inside typical feedback controller bandwidths. Such conditions imply the very action of sending control signals to the ultralight weight robotics will cause structural resonance. Implementations of incrementally increasing order are offered, achieving over ninety percent performance improvement in trajectory tracking errors, while improvement using unshaped methods merely achieve twenty-four percent improvement in direct comparison (where the only modification is the proposed control methodology). Based on superior performance, single-sinusoidal trajectory shaping is recommended with a corollary benefit of preparing future research into applying deterministic artificial intelligence whose current instantiation relies on single-sinusoidal, autonomous trajectory generation.
\end{abstract}

Keywords: robotics; monitoring; repair; replace; infrastructure; space robotics; flexible; structuralcontrols interaction; trajectory shaping

\section{Introduction}

This manuscript describes current novel methods in space robotics in the context of former methods inspiring paths for future ground-breaking discovery in the maintenance of on-orbit space infrastructure. Modern state of the art is compared to classical methods and recently proposed methods, but furthermore future novel methods are inspired and grounded in a basis of lessons learned in the comparisons of classical and modern methods.

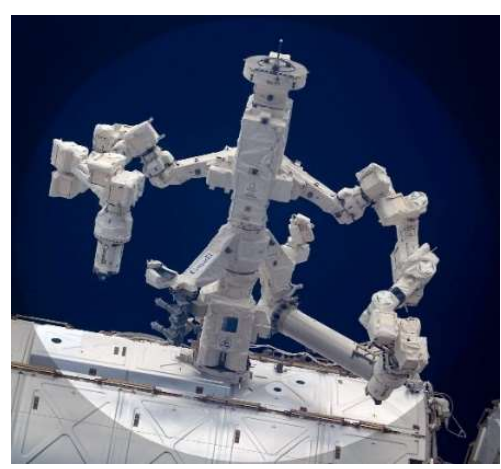

(a)

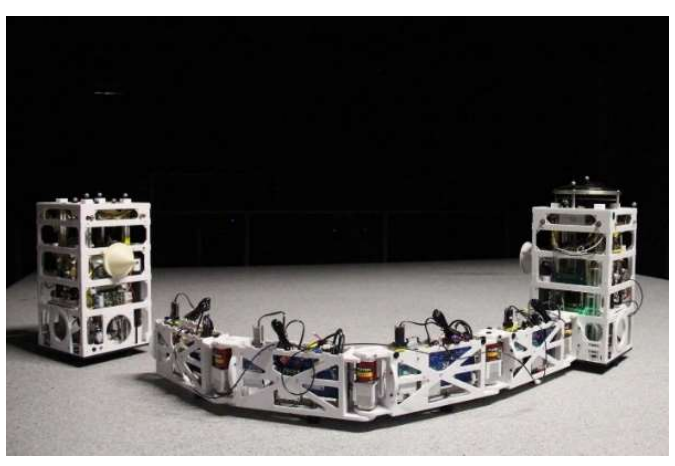

(b)

Figure 1. (a) NASA's remote-controlled robotic refueling mission on-orbit. [1] (b) U.S. Naval Postgraduate School's autonomous robotic laboratory free-floating simulator on planar air bearing table. [2]

1.1 Broad context and highlighting importance. 
Currently, placing material into low earth orbit costs roughly ten-thousand U.S. dollars per pound. [3] One key lifetime limitation is the finite quantity of fuel brought to orbit. [3] Other lifetime limitations include gradual degradation of solar panels reducing electrical power generation, and failure of other spacecraft components (e.g., electrical devices like radios, gradual decay of moving parts like gyroscope bearings, etc.). [4] An attractive remedy that is currently an active area of research includes in situ resource replenishment and requisite disassembly and assembly (construction) as depicted in figure 1a. [5] Benchboard spacecraft robotic laboratories (in figure 1b) [6] are useful to validate advanced thinking on the basic scientific aspects of possibilities of future operations establishing a functional supply chain of logistical support to spacecraft on-orbit.

\subsubsection{Modeling}

Modeling space robots (like those depicted in figure 2) from first principles articulated by Chasle [7] begins with application of Newton's second law of motion [8] for translation and Euler's moment equations [9] for rotation. Utilizing the finite element method, the robot may be discretized into a chosen number of nodes for application of these first principles establishing a system of differential equations whose order is drive by the chosen number of nodes.

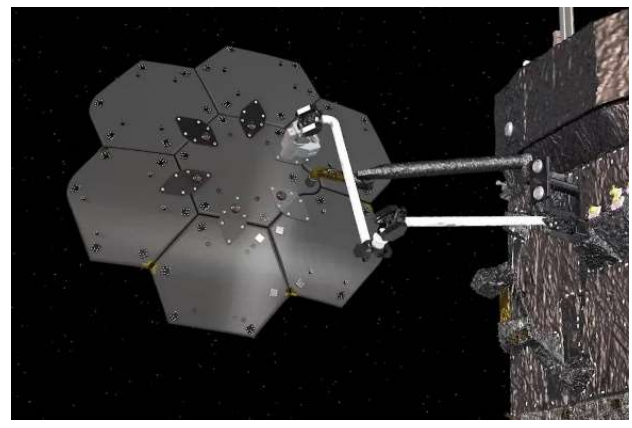

(a)

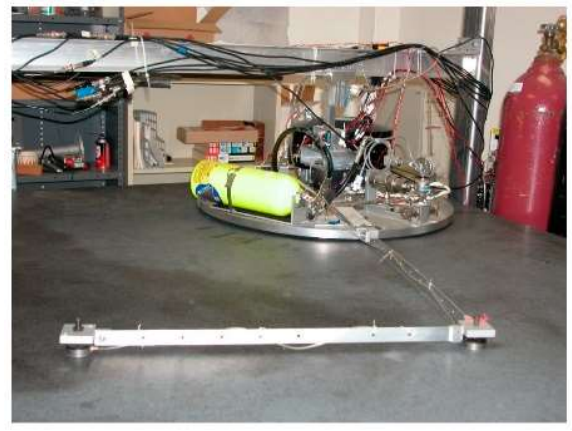

(b)

Figure 2. Robotic assembly and manufacturing in space (a) NASA and Maxar Technologies SPIDER (planned) robotic assembly in space. [5] (b) autonomous robotic laboratory free-floating simulator on planar air bearing table. [6] This testbed is the subject of modeling and control efforts leading to the discovery of whiplash compensation of flexible space robotics. [6]

\subsubsection{Classical methods}

Traditionally, the nonlinear coupled motion terms in both translation and rotation equations are neglected, linearized, or simplified by assumptions, permitting classical treatment of linear, time-invariant methods. [10] The treatment begins with gain stabilization through feedback and may be augmented with frequency-dependent filtering to shape the frequency-response of the robotic control attempting to negate the deleterious effects of structural flexibility.

\subsubsection{Modern methods}

The term "modern" is often used to either indicate problem formulation in spacevariable form also called "state space" form, [11-12] or alternatively deterministic optimization (as opposed to stochastic optimization commonplace in classical methods). [13] Modern methods, even optimal instantiations ubiquitously impose a specified limited form of the robotic control equation. Typically, the control is forced to be a negative feedback or errors, where gains associated with each error are discerned to optimize some specified performance function. One very common form of a modern optimal feedback controller is the linear quadratic regulator [14] (amongst others including robust L1 adaptive control [15-16]). An alternative modern method eliminated the mandatory use of feedback to discern a feedforward (open loop) approach to control flexible robotic systems 
where the deleterious responses of flexible, multi-body motion are codified in a method called input shaping [17-18] where anticipated strain energy is distributed with time to create a time-delayed control approach for flexible space robotics. [19] The methods (feedforward and feedback) have also been combined. [20]

\subsubsection{Recently proposed methods}

Post-modern methods eliminate the assertion of the presumed negative feedback form of the control with variable gains. The differential equations mentioned in section 1.1.1 may be expressed in vector-matrix form establishing dynamic constraints to form a time-varying, nonlinear Hamiltonian system [21] that may be optimized in accordance with the methods of Pontryagin. [22] The result of this approach applied to highly flexible space robotics is the recently revealed whiplash compensation of flexible space robotics. [23] The method was developed on a university benchboard free-floating space robot simulator depicted in figure $2 \mathrm{~b}$ representing such high-end missions as the NASA/Maxar robotic assembly mission (depicted in figure 2a) currently under construction. Whiplash compensation as proposed is an open-loop optimal approach (akin input shaping) focused on shaping the control to optimize rotational motion of highly flexible space robots. The unexpected counter-steering devised inspired renewed focus on shaping desired trajectories in the context of optimized controls leading to the just proposed deterministic artificial intelligence. [24]

\subsubsection{Potential methods}

The open loop optimal shaping of the commanded trajectory in addition to the unexpected results of nonlinear constrained optimization embodied in whiplash compensation inspire study of alternative trajectory shaping options. This manuscript will propose sinusoidal trajectory shaping options [25] to provide methods to steer the control signal's frequency content away from problematic frequencies. This investigation is inspired by the very recently proposed deterministic artificial intelligence method [24] utilizing sinusoidally shaped input trajectories. Especially since the newly proposed method is parameterized in terms of time-variant mass and mass moment of inertia, [26,27] the potential for fruitful application to autonomous space robotics seems high since the application requires operations amidst sudden increases and decreases in mass and mass moments. This potential is proposed for future research permitting deterministic artificial intelligence to be applied to highly flexible space robotics.

\subsection{Statements of novelty}

This paragraph succinctly states the novel contributions offered in the manuscript.

1. Trade-off study: compensate only for purposes of insuring stability versus compensation to flatten the frequency response curve enhancing regular predictability with regards to frequency of excitation.

2. Direct comparison of classical methods and a smoothed version of recently proposed whiplash compensation (originally proposed with non-asymptotically smooth ramp trajectories). [23]

3. Additional direct comparison with the newly proposed single-sinusoidal trajectory limitation currently the baseline approach for deterministic artificial intelligence methods.

4. Elaboration of future research using recently proposed deterministic artificial utilization of (autonomously generated) single-sinusoidal trajectory.

\subsubsection{Trade-off study on application of classical methods}

Classical proportional plus integral plus derivative control is design to satisfy nominal performance specifications (e.g., rise time, overshoot, settling time, etc.). Following the well documented methods of flexible system compensation. Second order filters are sequentially applied to each flexible mode: notch filtering of resonant response and bandpass filtering of anti-resonant responses associated with free-free vibrations of on-orbit 
space robots. Classical procedures primarily focus on portions of flexible modes (either resonance or anti-resonance) that influence system stability by generation of cross-over frequencies. Accordingly, only a single notch or bandpass filter is commonly used, which also helps limit system order, since addition of second order filters in the feedback path increases closed-loop system order. Trade-off studies are performed evaluating both the performance and increase in system order for progressively more structural filtering, eventually seeking to "flatten the curve" (the magnitude response plots) essentially nullifying flexible affects. While predictable response can be tuned to desired performance specification, these classical methods suffer from limited robustness to variation in system natural frequencies. Iterative application of classical structural filtering as instantiated in this research realizes eleven to four-hundred twenty percent improvement in gain margin and minus five to sixty-four percent improvement in phase margin with up to twentyfour percent improvement in tracking error in response to step commands. On the downside, the methods increase system order up to fifty implying high increase in computational burden.

1.2.2. Trade-off study of modern simple trajectory shaping methods and post-modern whiplash compensation. [23]

Trajectory shaping (input shaping, adaptive input shaping, zero-vibration and zerovibration derivative shaping, etc.) are recently proposed methods seeking to mitigate the deleterious effects of step inputs which theoretically exhibit infinite bandwidth. [28, 29] While predictable response can also be tuned to desired performance specification (as with classical structural filtering), these methods also suffer from limited robustness to variation in system natural frequencies and are mentioned here contextually, but not included in analytic treatment. Most recently whiplash compensation has been proposed in the literature, [23] and direct comparison is made in this manuscript.

\subsubsection{Proposed trajectory generation}

The aforementioned techniques use system analysis to identify natural frequencies with modeling, and then design controls based on the identified frequencies inherently limiting robustness when natural frequencies are known, poorly modeled, or time-variant.

An alternative paradigm is proposed here, instead designing controls based on one single frequency stemming from desired speed of response, and then ensuring that single frequency is well outside the possible proximity of system natural frequencies.

The proposed method accepts step commands by autonomously converting the commands into state trajectories based on single frequency sinusoids as proposed in recent publications on deterministic artificial intelligence. $[24-25,27]$ The proposed methods are implemented on four systems of incrementally increasing order, achieving over ninety percent performance improvement in trajectory tracking errors with step inputs, while improvement using unshaped methods merely achieve twenty-four percent improvement in direct comparison (where the only modification is application of the proposed methodology).

\subsection{Main aims of the manuscript}

The goal is to lay the groundwork from classical methods, through modern methods to post-modern methods to inspire and recommend future basic scientific research in autonomous space robotics permitting a single robotic inspection, replenishment and assembly robot system to service satellite with very disparate connections (figure 3) that were not designed for in-situ replenishment and assembly operations as depicted in figure 4's remotely controlled prototype for the suggested autonomous system. 


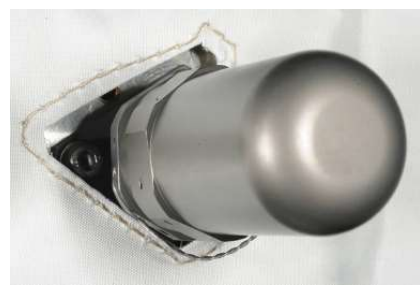

(a)

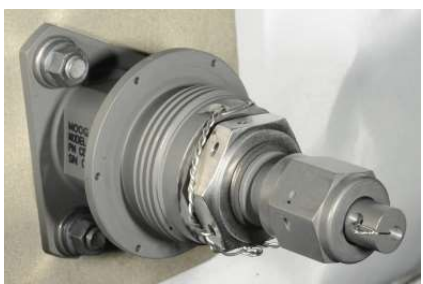

(b)

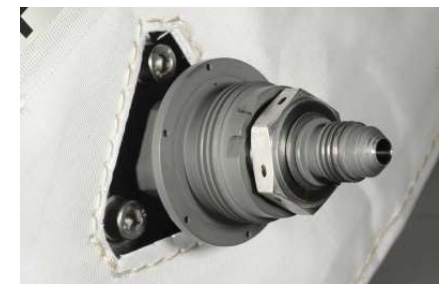

(c)

Figure 3. Disparate satellite fuel valves illustrating a challenging aspect of refueling an arbitrary spacecraft on-orbit (a) tertiary cap with "lock wire" visible underneath (b) safety cap/actuation nut with securing lock wire, and (c) exposed fuel valve. [3]

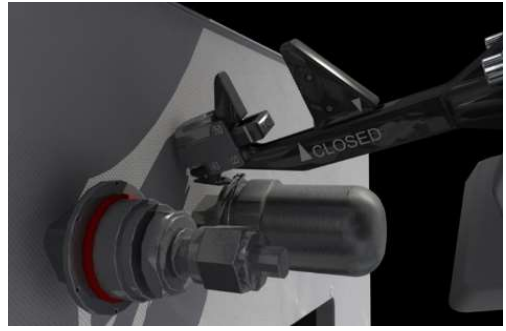

(a) Start with wire [3]

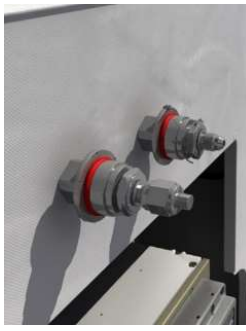

(b) [3]

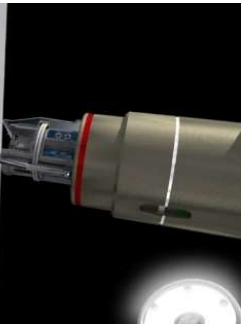

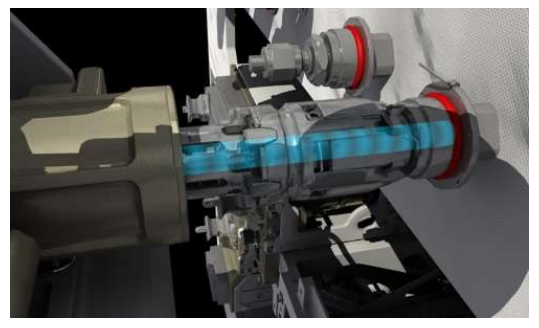

(b) [3]

Figure 4. Operations required to connect to various satellite fuel valves illustrating a challenging aspect of refueling an arbitrary spacecraft on-orbit (a) start with wire cutting; (b) connect nozzle tool with hose and transfer fuel; (c) leave behind quick-disconnect when re-fueling complete (for future refueling operations).

\section{Materials and Methods}

Modeling and modal system identification followed by control by focusing on development of the decision and control criteria, development and shaping or the desired (commended) input trajectory, and combinations of both shaping and control developments. This foundation leads to an instinct that parameterization methods that include time-varying mass and mass moment of inertia can be developed to mitigate rapid increases and decreases associated with grasping and releasing target-spacecraft of unknown or assumed poorly known mass properties. The suggested path for future basic scientific research is deterministic artificial intelligence recently developed for autonomous unmanned underwater vehicles. [24]

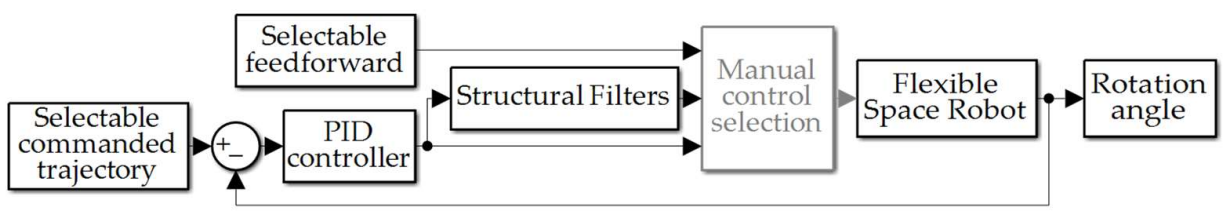

(a)

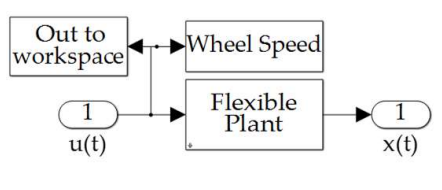

(b)

Figure 5. (a) Simulations created in SIMULINK used to make comparison of disparate approached (b) Flexible space robot subsystem depicted in subfigure (a).

Modeling is illustrated to be a combination of mathematical modeling [30-31] of rigid bodies [32-33] foremost followed by augmentation of the rigid body model with flexible components (e.g., robotic manipulator arms). [11, 34-35] Together, the combined model will establish the flexible space robot depicted in the SIMULINK model in figure 5a. The "manual control selection" box contains manual routing switches permitting direct 
comparisons where the only variation is the method of controlling the highly flexible space robot. Analysis performed in this manner produce figures of merit for decision makers deciding which approach to use for autonomous control in a given situation. The manual switches allow iteration of the various approaches coded in boxes to the left of figure 5a: PID, structural filtering, selectable feedforwards, and selectable (shaped) commanded trajectories. Figure $1 \mathrm{~b}$ illustrates the contents of the flexible space robot subsystem in the far right-hand side of figure $5 \mathrm{a}$ and $5 \mathrm{~b}$.

\subsection{Modeling and analysis of flexible space robots}

Modeling highly flexible space robots begins with treatment of rigid bodies which subsequently have appended to them highly flexible robotic appendages, where maneuvering the body is accomplished by a spinning wheel [36] exchanging angular momentum with the body. [37] The addition of the flexible robotic appendages is accomplished by repeated application of rigid body treatments at a chosen number of nodes, where the nodes result from discretization of the continuous structure of the robotic appendage.

\subsubsection{Rigid body dynamics}

Michel Chasles's theorem [7] allows us to simply invoke Newton [8] and Euler's equations [9] to fully describe the six degrees of freedom of mechanical motion. [38]

Rigid body dynamics have a long, established heritage dating back to at least the seventeenth century in the developments of Newton, [8] the eighteen century with the developments of Euler, [9] and the nineteenth century consolidation of the two by Chasle [5] and a generalized treatment by Hamilton [30] while the measurement in rotating reference frames was expressed one year later by Coriolis. [31] Developments continued and accelerated throughout the twentieth century by Mertz [32], Whittaker [33], Church [34], Wright [35], Gray [36], Rose [37], and Greenwood [39], Goldstein [40] and others; in particular Kane who instantiated a new expressions in 1959 and 1961 [41-42], elaborated later in 1985 [43] and subsequently evoked in the twenty-first century by Roithmayer, et al. [44]. These relationships were very recently used together with Pontryagin's treatment of optimization [22] to reveal whiplash compensation for the flexible space robot depicted in figure $5 b$ [23] in addition to the development last year of the burgeoning field of deterministic optimization [24] that uses these relationships to formulate self-awareness statements that are augmented by either nonlinear adaptive feedback or optimum (in the 2norm sense) learning that uses a reparameterization of the same basic dynamic relationships.

The basic relationships relating force to acceleration (per Newton) and torques to angular acceleration (per Euler) are illustrated in equations (1) and (2) respectively where the quantities are measured relative to a non-rotating reference frame (called "inertial"), while their expression in coordinates of rotating reference frames are expressed in equations (3) and (4) respectively. Simply invoking the two relationships comprise Chasle's theorem.

$$
\begin{gathered}
\left.F\right|_{\text {inertial }}=\left.m a\right|_{\text {inertial }}=\left.m \dot{v}\right|_{\text {inertial }}=\left.m \ddot{x}\right|_{\text {inertial }} \\
\left.T\right|_{\text {inertial }}=\left.J \alpha\right|_{\text {inertial }}=\left.m \dot{\omega}\right|_{\text {inertial }} \\
F=m \ddot{x}+m \frac{d \omega}{d t} \times r^{\prime}+2 m \omega \times v^{\prime}+m \omega \times\left(\omega \times r^{\prime}\right) \\
T=J \dot{\omega}+\omega \times J \omega
\end{gathered}
$$

Equations (3) and (4) are implemented in this manuscript as the rigid body mode of a flexible space robot designed for in situ replenishment or assembly operations on orbit where:

$F$ is the vector sum of the external forces acting on the body.

$\omega$ is the angular velocity. 
$v^{\prime}$ is the velocity relative to the rotating reference frames.

$r^{\prime}$ is the position vector of the object relative to the rotating refence frame.

$a^{\prime}$ is the acceleration relative to the rotating reference frame.

$m \frac{d \omega}{d t} \times r$ is the Euler acceleration.

$2 m \omega \times v^{\prime}$ is the Coriolis acceleration.

$m \omega \times\left(\omega \times r^{\prime}\right)$ is the centrifugal acceleration.

Paragraph 2.1.1 indicates the same results were obtained by Hamilton. Hamilton's principle implies that the Lagrange method must:

Minimize $J[x(g), u(g), t]=\int_{t_{0}}^{t_{f}} L(\dot{x}, x) d t$

Subject to $\dot{x}=u$

Where

$$
L=T-V=\frac{1}{2} m V^{2}+m g h=\frac{1}{2} m \dot{x}^{2}+m g h
$$

Writing the control Hamiltonian: $H(\lambda, x, u) \equiv L(x, u)+\lambda u$

Pontryagin's minimization condition: $\frac{\partial H(\lambda, x, u)}{\partial u}=\frac{\partial L(x, u)}{\partial u}+\lambda=0$

Differentiating leads to a minimization equation expression that may be combined with the Adjoint equation to produce the Euler-Lagrange equation, which by algebraic manipulation becomes Newton's Law.

$$
\begin{gathered}
\frac{d}{d t}\left(\frac{\partial L(x, u)}{\partial u}+\lambda\right)=\frac{d(0)}{d t}=0 \rightarrow \underbrace{\frac{d}{d t}\left(\frac{\partial L(x, u)}{\partial u}\right)-\frac{\partial L(x, u)}{\partial x}}_{\text {Euler-Lagrange equation }}=0 \\
\frac{d}{d t}\left(\frac{\partial L(x, u)}{\partial u}\right)-\frac{\partial L(x, u)}{\partial x}=\frac{d}{d t}\left(\frac{\partial L(x, u)}{\partial u}\right)-\frac{\partial L(x, u)}{\partial x}=\frac{d}{d t}(m \dot{x})-\frac{\partial L(x, u)}{\partial x} \\
=m \ddot{x}-\frac{\partial L(x, u)}{\partial x}=0 \\
\underbrace{\frac{\partial L(x, u)}{\partial x}}_{F}=m \underbrace{\ddot{x}}_{A} \Leftrightarrow \underbrace{F=m A}_{\text {Newton's Law }}
\end{gathered}
$$

where:

$H(\lambda, x, u)$ is the Hamiltonian.

$L(x, u)$ is the Lagrangian.

$\lambda$ is the nomenclature for the adjoints or costates associated with each state.

Hamilton's principle of minimizing the Lagrangian results in two methods to derive equations of motion: Newton's Law and the Euler-Lagrange Equation both represented by the so-called "double-integrator" (equations (1) and (2)) initially until elaborated by expression in rotating reference frames (equations (3) and (4)).

The three degrees of freedom of translational motion represented in equations (1) and (3) together with the three degrees of freedom of motion of rotation in equations (2) and (4) together completely specify six degrees of freedom ("6-DOF") of mechanical motion. The cross products of motion in equations (3) and (4) create a high level of complication, such that a general solution of this differential equation without simplifying assumptions remains unsolved.

Assuming feedback control whose signal is restricted to gains, $\mathrm{K}$ multiplied by state errors, controlling the rigid body equations of motion begin with the so-called double integrator relationships for both translation and rotation are often represented by spring- 
(a)

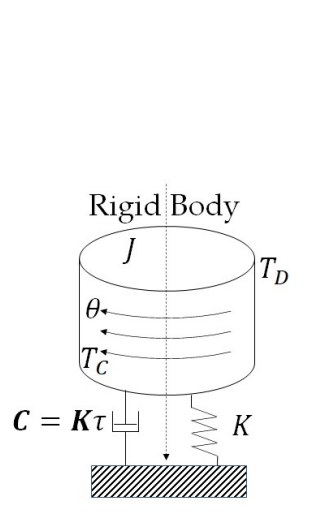

mass-damper system as depicted in figure $6 a$ whose response magnitude and phase may be plotted on log-normal graphs as depicted in figure $6 \mathrm{~b}$. The presence of two integrators in the response function numerator dominates the low frequency response with a response reduction by forty decibels per decade of frequency up to the breakpoint designated by the Greek letter omega, $\omega$ after which the response reduces 20 decibels per decade. A higher number of integrators in the denominator is called a "pole excess" and the high frequency phase lag asymptotically approaches this pole excess with increasing frequency.

Figure 6. Modeling and analysis of rigid bodies with respect to frequencies (a) modeling rigid bodies as systems with springs, masses (and mass moments of inertia), and dampers. (b) Frequency response analysis of the rigid body portion (or "rigid body mode") of the flexible space robot. (c) Frequency response analysis of the flexible body portion of the space robot depicted in figure $7 \mathrm{~b}$ (note 5 natural frequencies coresponding to the selection of five nodes, while the referenced system has also been modeled using eight nodes as displayed in figure 2 of [23]).

The seemingly simplified rigid body mode becomes the core of the flexible system in its labeling as the "rigid body mode" of the total flexible space robot. To complete the modeling effort, flexible parts are modeled separately and appended to the rigid body.

\subsubsection{Flexible body dynamics}

Discretizing the flexible appendages into a chosen number of nodes (four nodes used here illustratively as depicted in figure $7 \mathrm{~b}$ ) and rotational and translational equations from section 2.1.1 are repeatedly applied at each of the chosen nodes resulting in a system of equations of dimension equal to the chosen number of nodes.

The system of equations expresses in three axes is parameterized in accordance with the single ( $\hat{z}$-axis) rotation depicted in figure 8 a representing the flexible space robot simulator depicted in figure $8 \mathrm{~b}$. Notice the end effector manipulator position in this illustration is achieved by control of the attitude of the central rigid body. A neglected alternative is to bend the robotic arm using piezo-electric actuators as depicted in figure 10. Also omitted here is the grasping action of the robotic gripper (depicted in figure $7 \mathrm{~b}$ ). The rigid spacecraft hub in figure 8 is parameterized in reference frame (black font $\hat{x}, \hat{y}, \hat{z}$ ) centered at point frame $O$, which rotates an angle $\theta$ to angular positions designated by the red dashed line with red font $(\hat{X}, \hat{Y}, \hat{Z})$, while the actuator wheel (purple font $\hat{x}, \hat{y}, \hat{z}$ ) centered at point frame $O_{W}$, which rotates an angle $\theta_{W}$ to angular positions designated by the purple dashed lines with purple font $\left(\hat{x}^{\prime}, \hat{y}^{\prime}, \hat{z}^{\prime}\right)$. The vector of multi-dimensional forces $F$ on the robotic arm are decomposed into radial $\overline{\mathrm{r}}_{\mathrm{F}}$ and normal $\overline{\mathrm{u}}_{\mathrm{F}}$ components. 


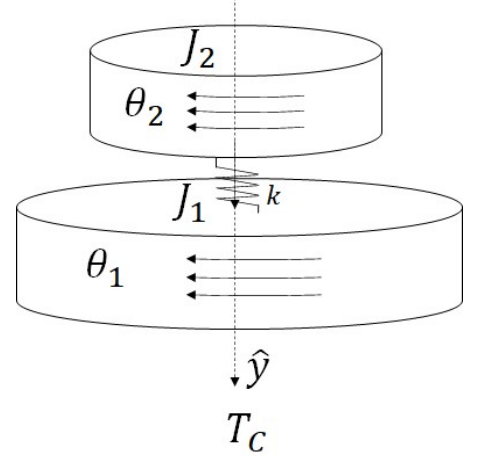

(a)

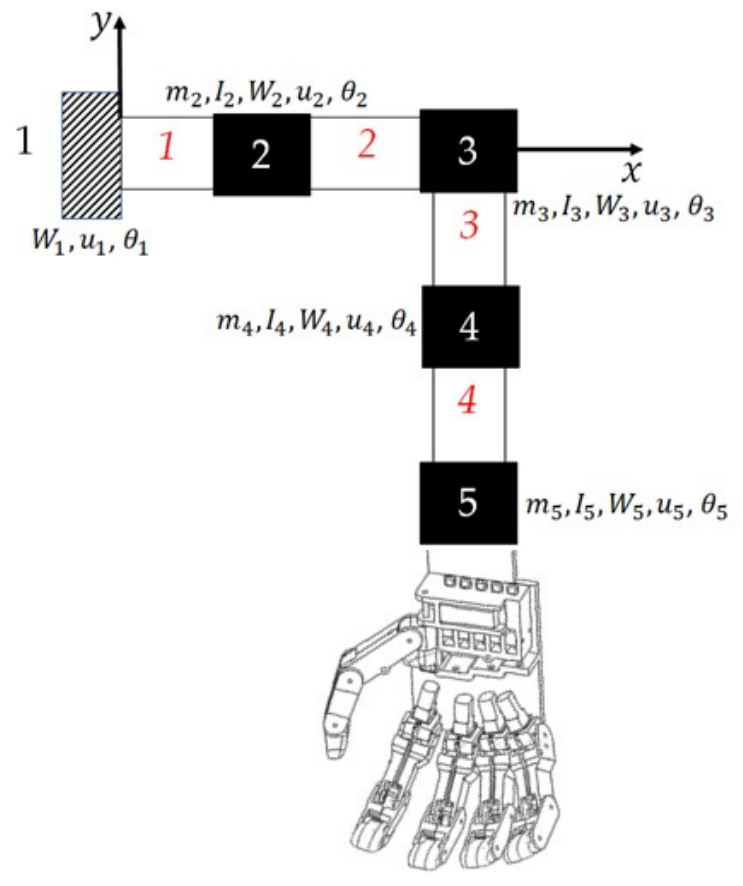

(b)

Figure 7. Modeling and analysis of flexible bodies discretized into four nodes depicted in (b) of mass moment of inertias $I_{2}$ through $I_{5}$ appended to the rigid body depicted in (a) as $J_{1}$, where $J_{2}$ in (a) represents the sum of constituent inertias at nodes $2-5$ in (b).

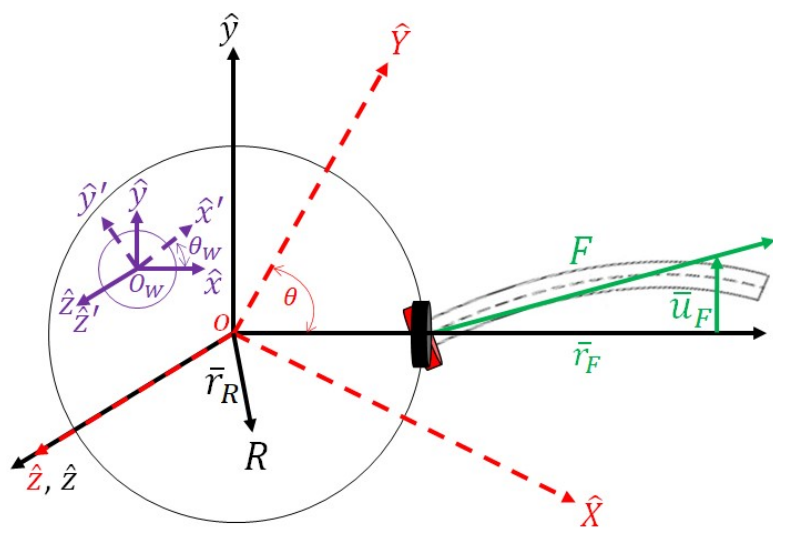

(a)

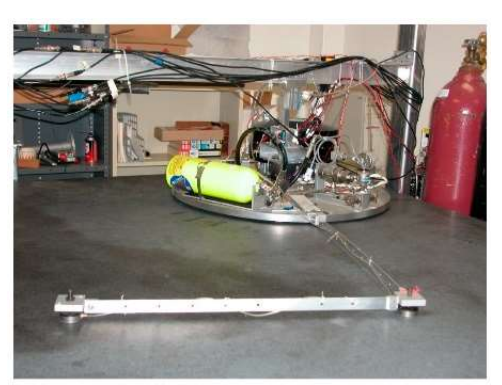

(b)

Figure 8. Schematic representation (a) of the flexible space robot depicted in figure 7 representing the free-floating flexible robot simulator at the U.S. Naval Postgraduate School depicted in (b).

Equations of Motion for the flexible system (rigid body plus flexible appendages may be conveniently derived using Euler-Lagrange's Method. Lagrange's method is appropriate regardless of various coordinate systems since it utilizes the systems kinetic (T) and potential energy $(\mathrm{V})$ in equation (13) to derive the equations of motion.

$$
T=\frac{1}{2}=\int_{R}\left(V_{R} \cdot V_{R}\right) d m+\int_{F}\left(V_{F} \cdot V_{F}\right) d m+\int_{W}\left(V_{W} \cdot V_{w}\right) d m
$$

where $\quad V_{R}=\dot{\theta} \hat{k} \times r_{R}$ is the velocity of a particle on the rigid body.

$V_{F}=\dot{\theta} \hat{k} \times r_{F}+\delta+\dot{\theta} \hat{k} \times \delta$ is the velocity of a particle on the flexible body

$V_{W}=\left(\dot{\theta}+\dot{\theta}_{W}\right) \hat{k} \times r_{W}+\dot{\theta} \hat{k} \times r_{o}$ is velocity of a reaction wheel particle 
$r$ vectors are position vectors to the respective particles

$\delta$ is elastic deformation vectors of particles on the flexible body.

$\hat{\imath}, \hat{\jmath}$, and $\hat{k}$ are unit vectors in the $\mathrm{x}^{\prime}, \mathrm{y}^{\prime}, \mathrm{z}^{\prime}$ axes of the reaction wheel.

Expanding all the terms in the equation for kinetic energy, $\mathrm{T}$ produces equation (14):

$$
\begin{gathered}
T=\frac{1}{2} \int_{R}\left(x_{R}^{2}+y_{R}^{2}\right) d m+\frac{1}{2} \int_{F}\left[\dot{\theta}^{2}\left(x_{R}^{2}+y_{R}^{2}\right)+\left(\dot{\delta}_{x}^{2}+\dot{\delta}_{y}^{2}\right)+\dot{\theta}^{2}\left(\delta_{x}^{2}+\delta_{y}^{2}\right)\right] d m \\
+\frac{1}{2} \int_{F}\left[2 \dot{\theta}\left(x_{f} \dot{\delta}_{y}-y_{f} \dot{\delta}_{x}\right)+2 \dot{\theta}^{2}\left(x_{f} \delta_{x}+y_{f} \delta_{y}\right)+2 \dot{\theta}\left(\dot{\delta}_{y} \delta_{x}+\dot{\delta}_{x} \delta_{y}\right)\right] d m \\
+\frac{1}{2} \int_{W}\left[\dot{\theta}^{2}\left(x_{0}^{2}+y_{0}^{2}\right)+\dot{\theta}^{2}\left(x_{w}^{2}+y_{w}^{2}\right)+2 \dot{\theta}\left(x_{0} x_{w}+y_{0} y_{w}\right)+\dot{\theta}_{w}^{2}\left(x_{w}^{2}+y_{w}^{2}\right)\right] d m \\
+\frac{1}{2} \int_{W}\left[2 \dot{\theta} \dot{\theta}_{w}\left(x_{w}^{2}+y_{w}^{2}\right)+2 \dot{\theta}_{w} \dot{\theta}\left(x_{0} x_{w}+y_{0} y_{w}\right)\right] d m
\end{gathered}
$$

Utilizing generalized coordinates $\theta, \theta_{W}, \delta$ assuming the reaction wheel rotates about its center of mass, and invoking the small displacement assumption simplifies the kinetic energy equation to equation (15):

$$
\begin{gathered}
T=\frac{1}{2} I_{z z} \dot{\theta}^{2}+\frac{1}{2} I_{w} \dot{\theta}_{w}^{2}+\frac{1}{2} I_{w} \dot{\theta} \dot{\theta}_{w}+\frac{1}{2} \int_{F}\left(\dot{\delta}_{x}^{2}+\dot{\delta}_{y}^{2}\right) d m+\dot{\theta} \int_{F}\left(\dot{\delta}_{x}^{2}+\dot{\delta}_{y}^{2}\right) d m \\
+\dot{\theta} \int_{F}\left(x_{F} \dot{\delta}_{y}+y_{F} \dot{\delta}_{x}\right) d m
\end{gathered}
$$

where mass moment of inertia with respect to the $\hat{z}$ axis is in equation (16)

$$
I_{z z}=I_{z Z}^{R}+I_{z Z}^{F}+I_{z Z}^{w}=\int_{R}\left(x_{R}^{2}+y_{R}^{2}\right) d m+\int_{R}\left(x_{F}^{2}+y_{F}^{2}\right) d m+\int_{R} d m+m_{w}\left(x_{0}^{2}+y_{0}^{2}\right)
$$

\subsection{Modal system identification}

Convert the elastic deformation $\delta$ into cantilever modal coordinates [12] using $\delta_{x}=\sum_{i=1}^{n} \phi_{i}^{x} q_{i}(t)$ and $\delta_{y}=\sum_{i=1}^{n} \phi_{i}^{y} q_{i}(t)$. This relationship describes the displacement at time $\mathrm{t}$ of a point at position $\mathrm{x}$ along the FSS is related to the mode shape $\phi$ ( $\mathrm{x}$ and $\mathrm{y}$ components) and the generalized coordinate or modal coordinate, $q_{i}(t)$ a sinusoidal function. The mode shape is a time-independent characteristic of the flexible structure. Using this relationship, kinetic energy $T$ in terms of generalized coordinates is equation (17).

$$
\begin{gathered}
T=\frac{1}{2} I_{z z} \dot{\theta}^{2}+\frac{1}{2} I_{w} \dot{\theta}_{w}^{2}+\frac{1}{2} I_{w} \dot{\theta} \dot{\theta}_{w}+\frac{1}{2} \int_{F}\left(\sum_{i=1}^{n} \sum_{i=1}^{n}\left[\phi_{i}^{x} \phi_{j}^{x}+\phi_{i}^{y} \phi_{j}^{y}\right] \dot{q}_{i} \dot{q}_{j}\right) d m+\dot{\theta} \int_{F}\left(x_{f} \sum_{i=1}^{n} \phi_{i}^{y} \dot{q}_{i}-y_{f} \sum_{i=1}^{n} \phi_{i}^{x} \dot{q}_{i}-\right) d m \\
T=\frac{1}{2} I_{z z} \dot{\theta}^{2}+\frac{1}{2} I_{w} \dot{\theta}_{w}^{2}+\frac{1}{2} I_{w} \dot{\theta} \dot{\theta}_{w}+\frac{1}{2} \sum_{i=1}^{n} \dot{q}_{i}^{2}+\dot{\theta} \sum_{i=1}^{n} D_{i} \dot{q}_{i}^{2} \\
\text { where rigid elastic coupling term } D_{i}=\int_{F}\left(x_{f} \phi_{i}^{y}-y_{f} \phi_{i}^{x}\right) d m
\end{gathered}
$$

Potential energy comes from stiffness and can be expressed in generalized coordinates as: $V=\frac{1}{2} \sum_{i=1}^{n} \omega_{i}^{2} q_{i}^{2} \quad$ where $\omega_{i}$ is the natural frequency of the $i^{\text {th }}$ mode. The virtual work is given [11] as: $\quad \delta W=\sum \boldsymbol{T} \delta\left(\theta+\theta_{w}\right)-\sum \boldsymbol{T} \delta \theta+T_{D} \delta \theta=\sum \boldsymbol{T} \delta \theta_{w}+T_{D} \delta \theta$ where $T_{D}$ is disturbance torques and $\sum T$ is total torques (not to be confused with $\mathrm{T}=$ kinetic energy). Revisiting the Euler-Lagrange equation, substituting potential and kinetic energy yields the equations of motion in terms of the generalized coordinates. 


$$
\begin{gathered}
\sum \boldsymbol{T}=I_{z z} \ddot{\theta}+\sum_{i=1}^{n} D_{i} \ddot{q}_{i} \\
\ddot{q}_{i}+2 \xi \omega_{i} \dot{q}_{i}+\omega_{i}^{2} q_{i}+D_{i} \ddot{\theta}=0
\end{gathered}
$$

Notice Newton's law results in the identical equations of motion accounting for the stiffness between adjacent nodes of the flexible robot appendage.

Newton's Law: $\sum F=m a$ applies at each node of the system, where coordinates are defined in the hybrid-coordinate system [12].

$$
\begin{aligned}
& m_{1} \ddot{x}_{1}=-k_{1} x_{1}+k_{2}\left(x_{2}-x_{1}\right) \\
& m_{2} \ddot{x}_{2}=-k_{2}\left(x_{2}-x_{1}\right)+F \\
& \underbrace{\left[\begin{array}{cc}
m_{1} & 0 \\
0 & m_{2}
\end{array}\right]}_{[M]} \underbrace{\left\{\begin{array}{l}
\ddot{x}_{1} \\
\ddot{x}_{2}
\end{array}\right\}}_{\ddot{x}}+\underbrace{\left[\begin{array}{cc}
k_{1}+k_{2} & -k_{2} \\
-k_{2} & k_{2}
\end{array}\right]}_{[K]} \underbrace{\left\{\begin{array}{l}
x_{1} \\
x_{2}
\end{array}\right\}}_{x}=\underbrace{\left\{\begin{array}{l}
0 \\
1
\end{array}\right\} F}_{\{F\}}
\end{aligned}
$$

$[M]$ is the system's global mass matrix (to be derived by finite element analysis)

$[K]$ is the system's global stiffness matrix (to be derived by finite element analysis)

$\{F\}$ is the force vector.

Including structural damping result in: $[M]\{\ddot{x}\}+[C]\{\dot{x}\}+[K]\{x\}=\{F\}$. The two degrees of freedom are $x / y$ displacements and $\theta$ rotations. $x / y$ rotations are mutually exclusive since we are not allowing plane stress \& plane strain. That means the members are not allowed to lengthen/shorten (bending displacements only). So, for the horizontal members, the $\mathrm{x}_{1}$ in the equation of Newton's Law corresponds to the $\mathrm{Y}$ direction bending displacements. Similarly, for vertical members, the $x_{1}$ corresponds to $x$-direction bending displacements. The nodal degrees of freedom are constrained to zero at the attachment point of the flexible and rigid bodies (forcing the appendage to stay attached). Also note that the corner node of the flexible body is permitted no $\mathrm{x}$ or $\mathrm{y}$ displacement degrees of freedom leaving only ๑ rotations. This reduces our anticipated [14x14] system to [12x12] eliminating the displacement degrees of freedom at the attachment point and corner. Translating Newton's Law into rotational form and adding the rigid-elastic coupling method results in:

$$
I_{z z} \ddot{\theta}+\sum_{i=1}^{n} D_{i} \ddot{q}_{i}+I_{w} \ddot{\theta}_{w}=T_{D}
$$

where $\quad I_{z z}=$ body principal moment of inertia with respect to $Z$-axis

$\ddot{\theta}=$ angular acceleration of the system rotation angle, $\theta$

$\mathrm{D}=$ rigid-elastic coupling term

$\ddot{q}=$ acceleration in generalized displacement coordinates

$I_{w}=$ reaction wheel principal moment of inertia with respect to $\mathrm{C}, \mathrm{Z}$ axis

$\ddot{\theta}_{w}=$ angular acceleration of the reaction wheel rotation angle, $\odot_{w}$

$T_{D}=$ disturbance torques

Torques may be combined to resemble the basic expression of Newton's Law more closely:

$$
I_{z z} \ddot{\theta}+\sum_{i=1}^{n} D_{i} \ddot{q}_{i}=\sum \boldsymbol{T}
$$

where $\quad T$ =sum of disturbance torques, and control torques of the reaction wheel.

Isolating the first term: 


$$
\ddot{\theta}+\frac{\sum_{i=1}^{n} D_{i}}{I_{z z}} \ddot{q}_{i}=\frac{\sum \boldsymbol{T}}{I_{z z}}
$$

and also note:

$$
\ddot{\theta}=\frac{\sum \boldsymbol{T}}{I_{z Z}}-\frac{\sum_{i=1}^{n} D_{i}}{I_{z Z}} \ddot{q}_{i}
$$

The implementation on the flexible space robot depicted in figures $2 b, 7$, and 8 is included in the appendix to aid repeatability. The natural frequencies and mode shape are nominal values (assuming accurate modeling). Time-varying natural frequencies driven by sudden changes in system mass remain a challenge that is motivation for the techniques proposed in this manuscript.

\subsubsection{Time-varying natural frequencies due to robot grasping.}

A unique complication of applying classical methods to robotics is the inherent necessity of grasping and releasing objects (typically of unknowable mass) modifying the rigid body mode potentially nullifying, or at least reducing the efficacy of the structural filters (to be described next). This facet of space robotics strongly motivates limiting the frequency content of the control command (as proposed in this manuscript) to avoid excitation of unknown new vibration modes. The time-varying mass also motivates investigation utilization of the recently published deterministic artificial intelligence that accounts for both time-varying robotic system mass and frequency-dependent input shaping to avoid the deleterious effects of robot flexibility.

\subsection{Frequency responses of step and periodic functions [24]}

The Fourier transform of a unit step function $f(t)$ as displayed in equation (28). Notice the impulse occurrence is summed with an extra term with variable $\omega$ resulting in the response containing all the frequencies. This complication implies utilization of step functions as input trajectories mandates subsequent actions to deal with the resonant response of exciting structural frequencies.

$$
f(t)=\left\{\begin{array}{ll}
1 & t \leq 0 \\
0 & t>0
\end{array} \leftrightarrow F(\omega)=\int_{-\infty}^{0} e^{-j \omega t} d t=\int_{0}^{\infty} e^{j \omega t} d t=\pi \delta(\omega)-\frac{1}{j \omega}\right.
$$

If you do a Fourier transform on the (time domain) function which is zero for all time before $t=0$ and 1 for all time after $t=0$, the result is an infinite, continuous spectrum of frequency components. The power of each component is, of course, infinitesimally low so you can't measure anything. It is a very idealized function and, as its (infinite) energy is spread over an infinite number of frequency components of infinitesimally small value. [29]

Meanwhile, noting the Fourier transform of the sine function in figure 9a compared to the frequency response function of the five-node space robot in figure $9 \mathrm{~b}$. The challenge is much easier to surmount: to choose the fundamental frequency of the sine function to shape input avoiding the natural frequencies identified for the flexible space robot.
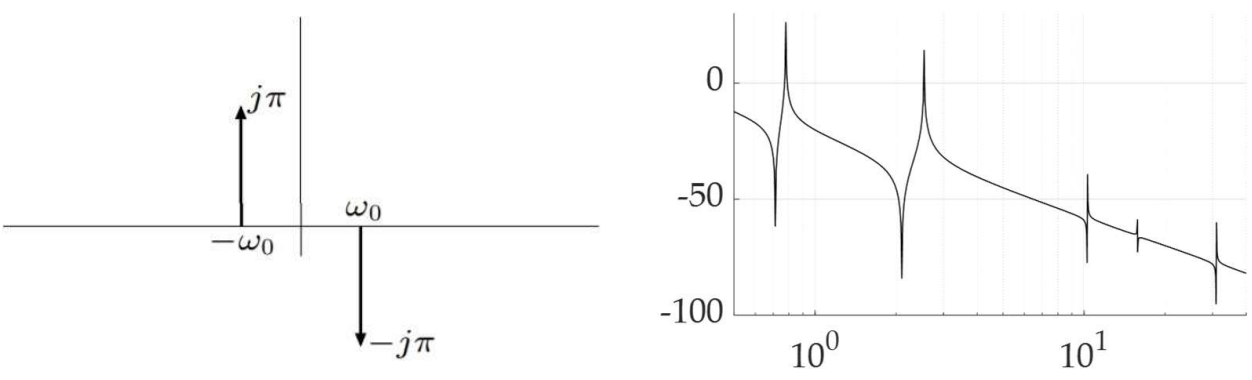
(a)

(b)

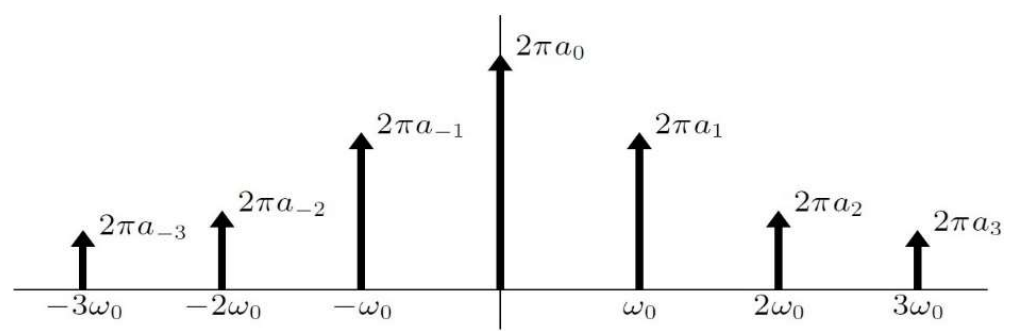

(c)

Figure 9. Fourier transform of sine with frequency on abscissa and response amplitude on the ordinate (b) sample robot frequency response with frequency on the abscissa and response magnitude on the ordinate (c) Fourier transform of periodic signal with fundamental frequency $\omega_{0}$ with frequency on abscissa and response amplitude on the ordinate. Notice the relatively easier (than using step functions) task of placing $\omega_{0}$ in (a) at locations other than the locations of the resonant and antiresonant peaks of $(\mathbf{b})$. The task using harmonic signals is slightly more challenging due to the necessity to properly place $\omega_{0}$ and all the harmonics depicted in (c).

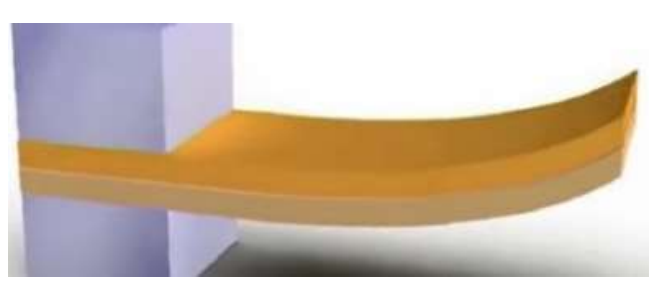

(a)

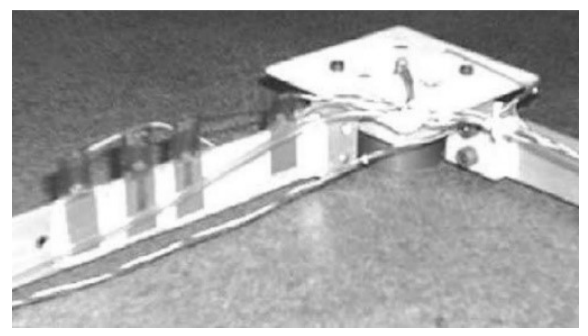

(b)

Figure 10. Piezo-electric materials may be used to control flexible robot arms. [45] (a) piezo bimorph bender; a kind of piezo-electric material used as both sensors and as actuators; $[46,47]$ (b) utilization of piezo-electric sensor and actuators utilized on the free-floating flexible space robot simulator in figure 8b [48] simulating hardware such as the Robotic Refueling Mission-3. [49] Subfigure b is taken from [47] a publication that is a work of the U.S. Government as defined in Title 17, United States Code, Section 101. Copyright protection is not available for this work in the United States.

\subsection{Alternative sensor and actuator options}

Due to the challenge of lofting pounds of weight into space, one alternative method of actuation utilizes very lightweight piezo electric materials. The placement of the endmost tip of the gripper in figure $7 \mathrm{~b}$ is proposed by manipulation of the rigid body central spacecraft hub. A neglected alternative introduced here for the sake of completeness is to mount piezo-electric materials along the lightweight links connecting the end effector to the rigid body spacecraft base. Figure 10a illustrates the result of sending electric current through piezo-electric materials. The robot link bends to a commanded shape manipulating the position and orientation of the end effector (gripper) within limits. Figure 10b illustrates some sample mounting positions of piezo-electric materials used as both sensors and actuators. [46,47] Figure 10c illustrates piezo electrics mounted on the robot links of the laboratory experimental hardware depicted in figure $8 \mathrm{~b}$ and modeled in figure $8 \mathrm{a}$ resulting in the model elaborated in Appendix A using the computer code included in Appendix $B$.

\subsection{Classical control approaches}

Rather than use of piezo electric actuators along the robot structure, classical control of the end effector is achieved by controlling the central spacecraft's rigid body hub. 
Presented in the following sections are several options for achieving such control: classical gain stabilization, classical structural filtering, modern time-delay input shaping, recently proposed whiplash compensation, presently proposed rigid-body optimal input shaping, and alternative proposed frequency shaping of inputs.

The implementation of the proposed frequency shaping of the inputs motivates the proposal of future research in the application of deterministic artificial intelligence, since the method is currently instantiated using the same frequency shaped inputs, and utilization of the method bestows the additional benefit of tracking time-varying natural frequencies (resonant and anti-resonant).

\subsection{Gain stabiliztion}

Gain stabilization begins by asserting a specified form of the feedback control. Both classical and modern (state space and optimal) control instantiations ubiquitously impose $u=-K x$ where the control is formulated as negative feedback of state errors multiplied by user-designed gains. Controllers with proportional plus integral plus derivative natures are called PID controllers, and they remain quite popular due to robustness, simple structure, ease of implementation, and continuing active research [50-52] in tuning the three gains associated with proportional, integral, and derivative components respectively. [53] Equation (29) illustrates the equations for a control with proportional plus velocity components whose resulting closed loop equation is displayed in equation (30) which may be expressed in terms of active damping, active stiffness, and feedforward in equation (31). Expression of the closed-loop system in Laplace domain as a transfer function is represented in equation (32).

$$
\begin{aligned}
& u=K_{p}\left(\theta_{d}-\theta\right)+K_{v} \omega \rightarrow J \dot{\omega}=T_{W} \equiv u(t) \\
& I \ddot{\theta}+K_{V} \dot{\theta}+K_{P} \theta=K_{P} \theta_{d} \\
& I \ddot{\theta}+\underbrace{K_{V} \dot{\theta}}_{\begin{array}{c}
\text { active } \\
\text { damping }
\end{array}}+\underbrace{K_{P} \theta}_{\begin{array}{c}
\text { active } \\
\text { stiffness }
\end{array}}=\underbrace{K_{P} \theta_{d}}_{\begin{array}{c}
\text { feed } \\
\text { forward }
\end{array}} \rightarrow\left[I s^{2}+K_{v} s+K_{p}\right] \theta(s)=K_{P} \theta_{d}(s) \\
& \frac{\theta(s)}{\theta_{d}(s)}=\frac{K_{p}}{I s^{2}+K_{v} s+K_{p}} \rightarrow \text { C.E. }: s^{2}+K_{v} s+\left.K_{p}\right|_{I=1}=s^{2}+2 \xi \omega_{n} s+\omega_{n}^{2}
\end{aligned}
$$

Gains may be designed foremost for stabilization, and additionally for performance specifications, e.g., rise time, peak overshoot, settling time, etc. While these specifications are met, residual oscillatory vibrations remain complicating on-orbit robotic operations. The transfer function expression particularly reflects the natural frequencies of the system, and these frequencies guide the subsequent steps in the classical approach: structural filtering to tailor the compensation at these frequencies.

\subsection{Classical second-order structural filtering}

The compensation of the system elaborated in equation (32) is often augmented by structural filters whose transfer function is represented by equation (33), where the frequencies of the zeros and poles ( $\omega_{z}$ and $\omega_{p}$ respectively) and damping of the zeros and poles $\left(\zeta_{z}\right.$ and $\zeta_{p}$ respectively) are designable and lead to the steady state gain, phase, and maximum gain are revealed in equations (34) - (36). Figure 11 illustrates how notch and bandpass filters respectively may be implemented by choice of the four designable parameters: $\omega_{z}, \omega_{p}, \zeta_{z}$ and $\zeta_{p}$

$$
\begin{gathered}
\frac{\operatorname{Output}(s)}{\operatorname{Input}(s)}=\frac{\frac{s^{2}}{\omega_{z}^{2}}+\frac{2 \zeta_{z}}{\omega_{z}^{2}} s+1}{\frac{s^{2}}{\omega_{p}^{2}}+\frac{2 \zeta_{p}}{\omega_{p}^{2}} s+1} \\
K_{\infty}=40 \log _{10}\left(\omega_{p} / \omega_{z}\right)
\end{gathered}
$$




$$
\begin{gathered}
\phi_{\text {max }}=\cos ^{-1}\left[\frac{\left(2 \zeta_{c} \sqrt{\omega_{p} / \omega_{z}}\right)^{2}-\left(\omega_{p} / \omega_{z}-1\right)^{2}}{\left(2 \zeta_{c} \sqrt{\omega_{p} / \omega_{z}}\right)^{2}+\left(\omega_{p} / \omega_{z}-1\right)^{2}}\right] \\
K_{\max }=20 \log _{10}\left(\zeta_{z} / \zeta_{p}\right) d B
\end{gathered}
$$

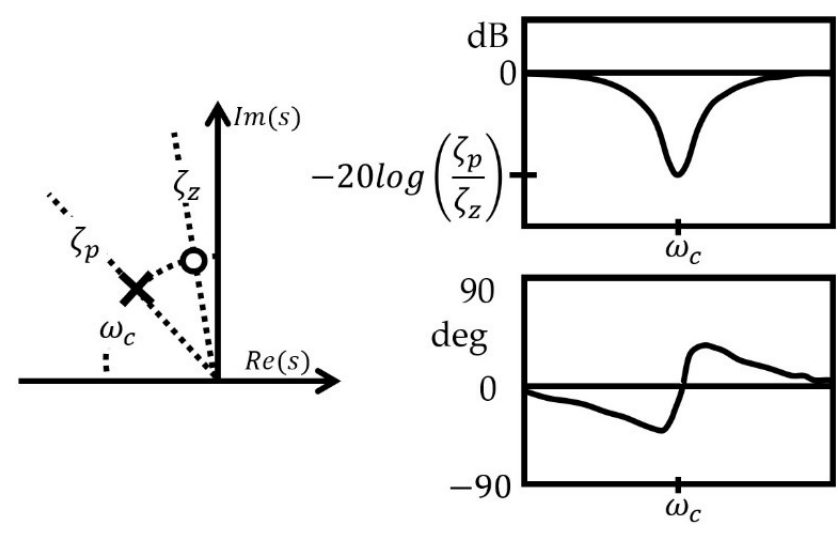

(a)

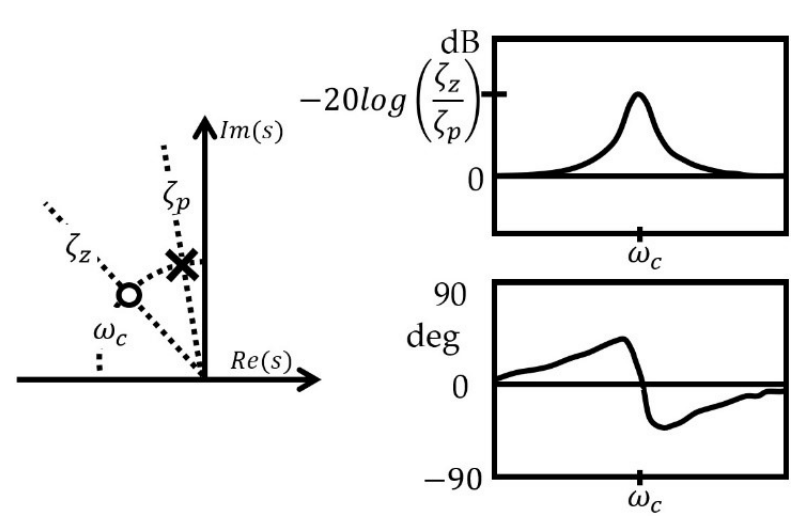

(b)

Figure 11. Depictions of second order structural filters. Graphics created by author duplicating depiction in [10] (a) Notch filter named after the nature to "notch" out the response surrounding specific frequencies (e.g., $\left.\omega_{C}\right)$. (b) Bandpass filter named after the nature to only the response surrounding specific frequencies (e.g., $\omega_{C}$ ). Filters are placed in series as depicted in figure 12.

$$
\begin{gathered}
\omega_{1} / \omega_{c}=\sqrt{2 \zeta_{z} \zeta_{p}+1-\sqrt{\left(2 \zeta_{z} \zeta_{p}+1\right)^{2}-1}} \\
\omega_{2} / \omega_{c}=\sqrt{2 \zeta_{z} \zeta_{p}+1+\sqrt{\left(2 \zeta_{z} \zeta_{p}+1\right)^{2}-1}}
\end{gathered}
$$

\subsubsection{Incremental application: ensure stability versus flatten the curve}

Subsequent application of filters expressed by equation (33) is displayed in figure 12 's depiction of the simulation blocks used to produce the results in section 3. A debatable design question is which filters are recommended for implementation. Utilization of both notch and bandpass filters at each identified node would seek to flatten the curve (the amplitude plot of the frequency response).

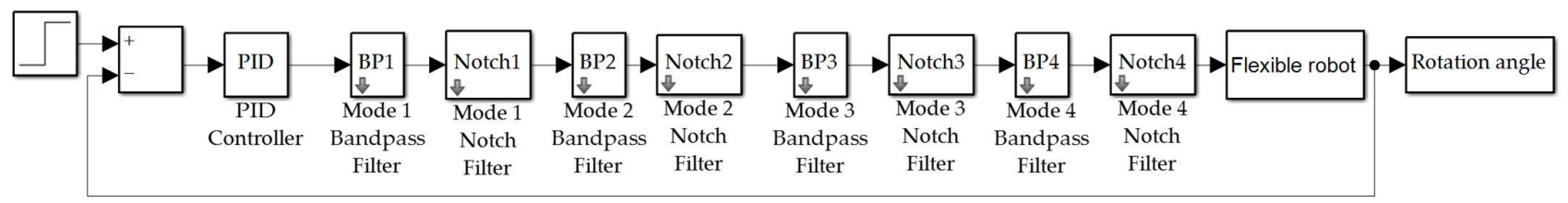

Figure 12. Topology of classical methods combining proportional, integral, derivative control with second-order structural filters (bandpass, abbreviated BP and notch) in accordance with equation (33) - (36) and the depictions in figure 13. Equations (25) - (27) may be used as the governing equations of motion for the flexible robot in the right hand side of figure 12.

Additional measures were investigated to control an assumably very highly flexible, lightweight space robot inspired by modern optimal open loop techniques like time-delay input shaping. A key strength is the avoidance of using step commands as input trajectories, so several methods were compared, replacing the step input with four alternatives depicted in figure 13b: modern time-delayed input-shaping, rigid body optimal input 
trajectory, recently proposed whiplash compensation, and the presently proposed single sinusoidal input trajectory, where the single sine is chosen for both speed of performance and avoidance of flexible resonance and anti-resonance depicted in figure $9 \mathrm{~b}$ and the dashed line in figure 13a. Each technique is described in the immediately following sections.

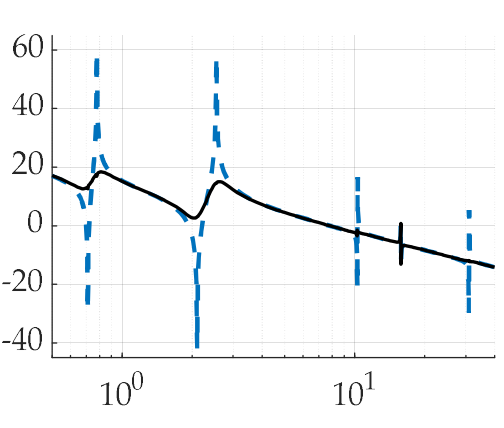

(a)

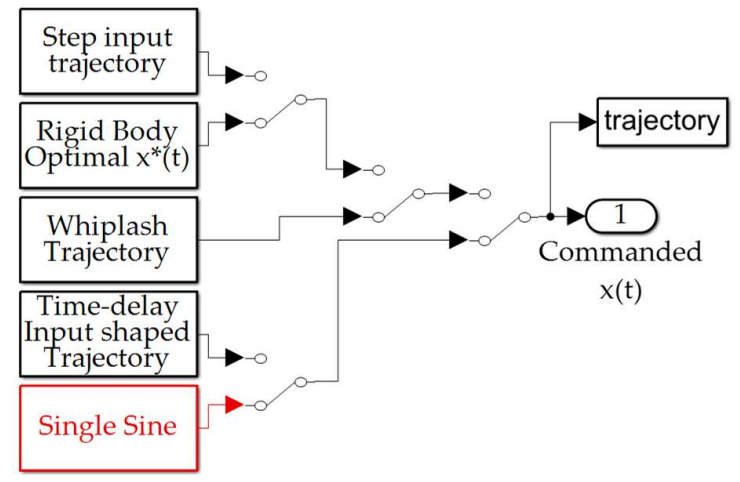

(b)

Figure 13. Compensation of multiple flexible modes of vibration (a) plot frequency response function with frequency (in radians per second) on the abscissa and response in decibels on the ordinate. The context of this manuscript's title is evident in this plot; (b) simulations created in SIMULINK to optionally switch between disparate methods facilitating direct comparison.

\subsection{Whiplash compensation input trajectory shaping}

So-called whiplash compensation was recently proposed [23] as the solution to constrained optimization problem of controlling highly flexible robotics with initial control in the opposite direction than the desired end state, subsequently implementing a ramp function held until the desired final time. The system studied in [23] is identical to that studied here, displayed in figure 8.

\subsection{Rigid body optimal input trajectory shaping}

Trajectory optimalization utilizing the methods of Pontryagin [28] is a competing option stemming from the just published [29] including nonlinear constrained optimization, real-time optimal control, and instantiations of such with and without singular switching and nonlinear decoupling of transport theorem terms. The intention in this manuscript is to establish a new benchmark for comparison of these just published methods in a proposed sequel.

\subsection{Input-shaping}

Input shaping is a technique that was state-of-the-art in the 1990's when the topic of flexible space robotics last saw a zenith where the method of time-delay was applied to the control signal, and the topic remains an active area of research. [54-56] The topic is considered citable and well-known in the context [57] and described here in just-enough detail to understand attempts to repeat this manuscript's research. Figure 14 illustrates the technique accompanied by a key quote cited at [58].

Input-shaping is open-loop control technique for reducing vibrations in computercontrolled machines. The method works by creating a command signal that cancels its own vibration. That is, vibration excited by previous parts of the command signal is cancelled by vibration excited by latter parts of the command. Input shaping is implemented by convolving a sequence of impulses, known as an input shaper, with any arbitrary command. The shaped command that results from the convolution is then used to drive the system. If the impulses in the shaper are chosen correctly, then the shaped command will excite less residual vibration than the unshaped command. 
The amplitudes and time locations of the impulses are obtained from the system's natural frequencies and damping ratios. Shaping can be made very robust to errors in the system parameters. [59, Chapter 5]

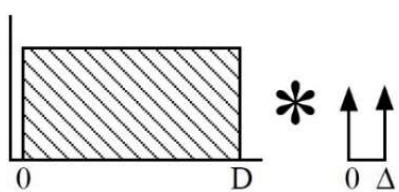

(a)

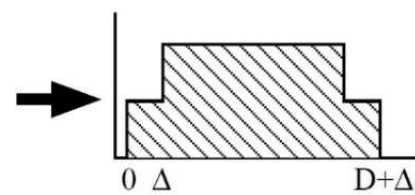

(b)

Figure 14. Convolution of initial command and shaper in subfigure (a) resulting in the shaped command in (b) where amplitudes and time locations $\mathrm{D}$ and $\Delta$ of the impulses depend on the system natural frequency and critical damping ratio $(\omega$ and $\zeta)$.

While the well-known implementation of input shaping addressed the commanded control signal, here the general notion of time-delay is applied to the desired trajectory, augmenting the notched and band-passed filtered controllers.

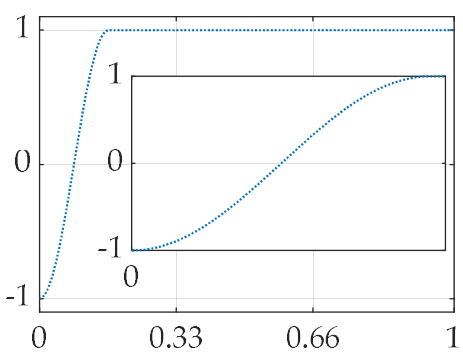

(a)

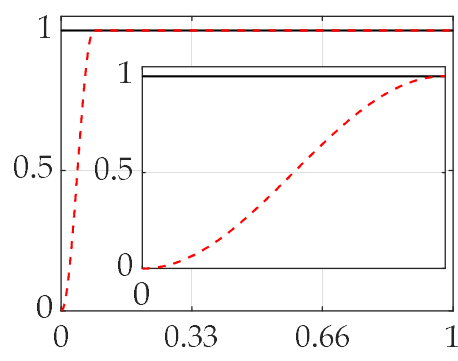

(b)

Figure 15. Alternative frequency trajectory shaping proposals (a) nonlinear, optimal whiplash trajectory shaping (b) single-frequency trajectory shaping. Notice the unexpected opposite initial direction of the commanded optimal "whiplash" trajectory.

\subsection{Single-frequency trajectory shaping}

So-called versine torque profiles [45] use smooth transcendental functions as a basis for "shaping" torque generation (like thew time-delayed input control shaping). With that inspiration, single-frequency sinusoids are proposed to instead shape autonomous trajectories by mimicking discontinuous step functions for flexible space robotics as depicted in figure 15. Two methods are displayed: 1) single-frequency implementation of whiplash trajectories, and 2) single-frequency shaping of step-like trajectories. Single frequency sinusoids are also the current state of the art trajectory shaper in deterministic artificial intelligence [24] making successful implementation in this manuscript foreshadow a likely sequel research effort to integrate optimized control of flexible space robotics with deterministic artificial intelligence.

\subsection{Deterministic artificial intelligence}

\subsubsection{Self-awareness statements}

The foremost premise of deterministic artificial intelligence is to assert physics-based [26] statement of self-awareness [27] comprised of the governing differential equations of motion (e.g. equations (35) - (38)) expressed in terms of desired trajectories [24] which must be analytic expressions to facilitate autonomous calculation. The desired trajectories are currently formulated using single sinusoidal trajectories [24] as described in section 2.11, while the current novel developments seek to utilize constrained optimal trajectories [60] in hopes of favorable comparison to sinusoidal trajectories. 


$$
\begin{gathered}
F=m \ddot{x}+m \frac{d \omega}{d t} \times r^{\prime}+2 m \omega \times v^{\prime}+m \omega \times\left(\omega \times r^{\prime}\right) \\
u \equiv m \ddot{x}_{d}+m \frac{d \omega_{d}}{d t} \times r_{d}^{\prime}+2 m \omega_{d} \times v_{d}^{\prime}+m \omega_{d} \times\left(\omega_{d} \times r_{d}^{\prime}\right) \\
T=J \dot{\omega}+\omega \times J \omega \rightarrow u \equiv J \dot{\omega}_{d}+\omega_{d} \times J \omega_{d} \\
I_{z z} \ddot{\theta}+\sum_{i=1}^{n} D_{i} \ddot{q}_{i}+I_{w} \ddot{\theta}_{w}=T_{D} \rightarrow u \equiv I_{z z} \ddot{\theta}_{d}+\sum_{i=1}^{n} D_{i} \ddot{q}_{i_{d}}+I_{w} \ddot{\theta}_{w_{d}}
\end{gathered}
$$

The modification of the governing differential equations of motion by replacing the motion states with desired states necessitates autonomous desired state trajectory generation, and this partially motivates the application of single-sinusoidal frequency-based state trajectories. Development of such trajectories in this manuscript make possible future sequel research applying deterministic artificial intelligence (as currently instantiated) to the optimal control of highly flexible space robotics.

\subsubsection{Adaption or optimal learning}

By reparametrizing equations (35) - (38) into regression form, optimal learning [27] or simple adaption [26] may be used to update the regression vector of unknowns (typically including variables such as mass, mass moments of inertia, and environmental properties such as those of the atmosphere, magnetic field, gravitational acceleration, and solar radiation.

\subsection{Simulation parameters}

MATLAB/SIMULINK release $2021 \mathrm{~b}$ was used to create simulations included in the appendices whose results are presented in section three. The Runge-Kutta integration solver was used with a fixed time-step of 0.01 seconds.

\section{Results}

This section provides a concise and precise description of the experimental results, their interpretation, as well as the experimental conclusions that can be drawn. Section 3.1 establishes a classical benchmark: gain stabilization using proportional, integral, derivative control. Section 3.2 augments section 3.1 by adding second-order classical structural filters (notch and bandpass sequentially). Filters are added incrementally in sections $3.2-$ 3.5 for each structural mode from low frequency to high frequency seeking to ascertain the minimal necessary instantiation, culminating in a comparisons of stability margins in section 3.6. Results using trajectory shaping are presented in section 3.7 followed by direct comparison in section 3.8 .

\subsection{Gain stabilization using proportional plus integral plus derivative control}

Gain stabilization is the established performance benchmark, and the results will appear in subsequent comparisons, incrementally adding additional compensation. Results are qualitatively compared to the benchmark in step response plots and frequency response plots with quantitative comparisons using gain margin and phase margin for stability and mean tracking errors for performance.

\subsection{Gain stabilization plus structural filters (bandpass $\mathcal{E}$ notch) for first flexible mode}

Gain stabilization was augmented with structural filters using equation (33) where structural resonant frequencies were used as filter natural frequencies and filter damping was adjusted to visually "flatten the curve" of the frequency response plot. First, a bandpass variant was designed for the first anti-resonant spike. Next, a notch variant was 
designed for the first resonant spike. Filter details are available in the appendix to aid repeatability. Figure 16 displays the results. Notice in the step response plot in subfigure (a) the nominal performances are nearly indistinguishable, while in the "inset" plot distinct differences are apparent, where quantitative results are displayed in Table 1 inserted as subfigure (b). Figure 17 displays four frequency response plots overlayed. The nominal frequency response (prior to gain stabilization) is lowest indicated by the dashed blue line. The thin-solid red line indicates gain-stabilized case. Meanwhile the overlaid dashed gold line indicates bandpass compensation of the first anti-resonance. Finally, the "flattened" frequency response plot's thin, dotted black line indicates full mode compensation with the addition of a notch filter at the first structural resonant frequency. Effects on frequency response phase is displayed in subfigure (b). Addition of the bandpass filter at the first anti-resonance leads to the highest gain margin with excellent phase margin, while addition of the notch filter at the first resonance increases phase margin substantially further with a slight decrease in gain stability.

\subsection{Gain stabilization plus structural filters (bandpass \& notch) for two flexible modes}

Gain stabilization with fully compensated first mode was augmented with structural filters using equation (33) where structural resonant frequencies were used as filter natural frequencies and filter damping was adjusted to visually "flatten the curve" of the frequency response plot at the location of the second mode. First, a bandpass variant was designed for the second anti-resonant spike. Next, a notch variant was designed for the second resonant spike. Figure 18 displays the results. Notice once again in the step response plot in subfigure (a) the nominal performances are nearly indistinguishable, while in the "inset" plot distinct differences are apparent, where quantitative results are displayed in Table 2 inserted as subfigure (b). Figure 19 displays four frequency response plots overlayed. The nominal frequency response (after gain stabilization) is indicated by the dashed blue line. The thin-solid red line indicates addition of full compensation of the first resonant mode. Meanwhile the overlaid dashed gold line indicates bandpass compensation of the second anti-resonance. Finally, the "flattened" frequency response plot's thin, dotted black line indicates full mode compensation with the addition of a notch filter at the first two structural resonant frequencies. Effects on frequency response phase is displayed in subfigure (b). Addition of both bandpass filter at the second anti-resonance and notch filter at the second resonance increases reduces both gain and phase margin substantially. Compensation of the second resonant structure mode should therefore be avoided, despite an instinct to "flatten the curve" of the frequency response plot magnitude. 


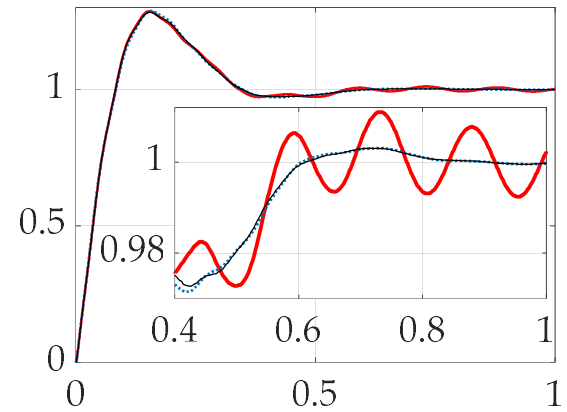

(a) PID (thick, solid red line); PID + bandpass 1 (dotted blue line); and PID + bandpass $1+$ notch 1 (thin, solid black line) illustrating substantial improvement by compensating for first anti-resonance, but negligible difference also compensating for first resonance.
Table 1. Compensation of rigid body plus first flexible mode (resonance and anti-resonance) with step input.

\begin{tabular}{cccc}
\hline System & $\begin{array}{c}\text { Gain } \\
\text { margin } \\
(\mathbf{d B})\end{array}$ & $\begin{array}{c}\text { Phase } \\
\text { Margin } \\
\text { (degrees) }\end{array}$ & Stable / Unstable \\
\hline Uncontrolled & 64.5 & 0.000673 & Stable \\
\hline PID controlled & 4.95 & 17.9 & Stable \\
\hline PID + bandpass & $-26.2^{1}$ & 80.2 & Stable \\
\hline PID + bandpass + notch & $-25.9^{1}$ & 133 & Stable \\
\hline
\end{tabular}

${ }^{1}$ Negative gain margins indicate that stability is lost by decreasing the gain, while positive gain margins indicate that stability is lost by increasing the gain.

Figure 16. Five computer simulations of control of rigid body; compensated first flexible anti-resonance, compensated first resonance, compensated second anti-resonance; and fully compensated first two modes with frequency in hertz on the abscissa and response magnitude in degrees on the ordinate.

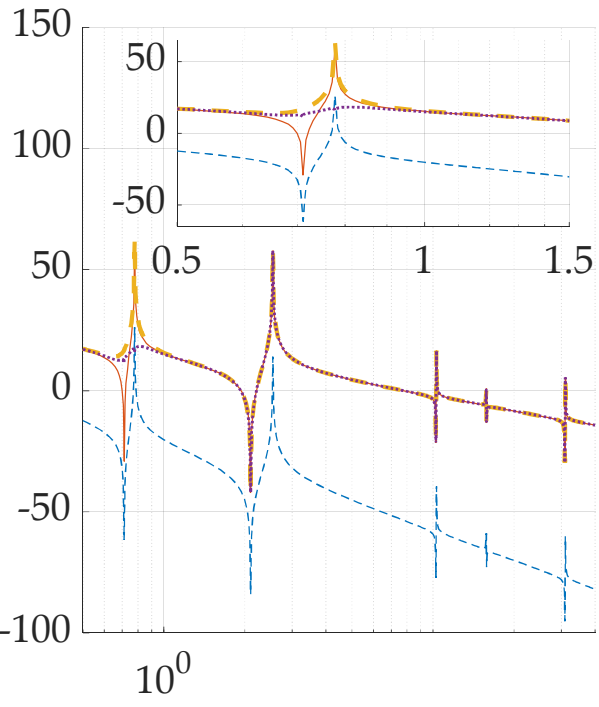

(a)

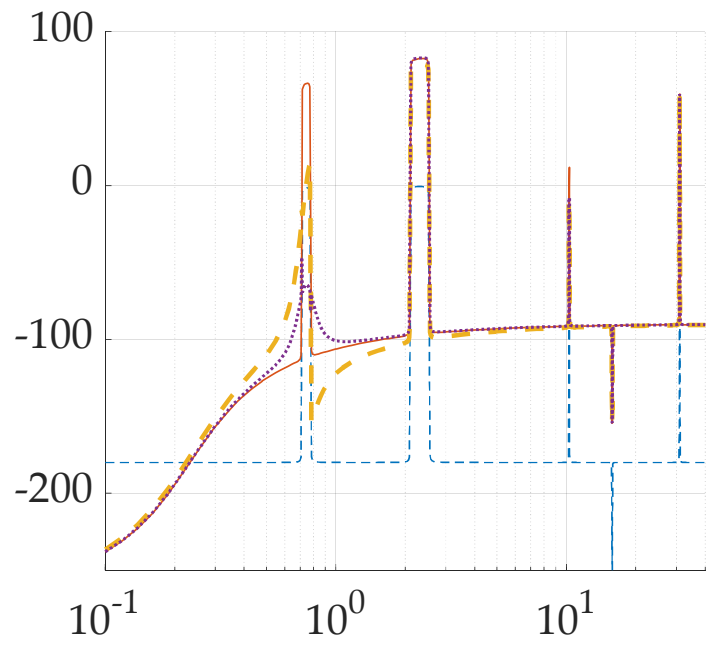

(b)

Figure 17. Four computer simulations of control of rigid body and first flexible mode with frequency in hertz on the abscissa: uncontrolled (narrow, dashed line), PID compensated (thin, solid line), PID compensated with bandpass filter of first antiresonance (thick, dashed line), and PID compensated with bandpass and notch filter of first mode - anti-resonance and resonance (dotted line) NOTE WATERBED EFFECT (a) Response magnitude (first mode zoomed in the inset) on the ordinate, (b) Phase in degrees on the ordinate. 


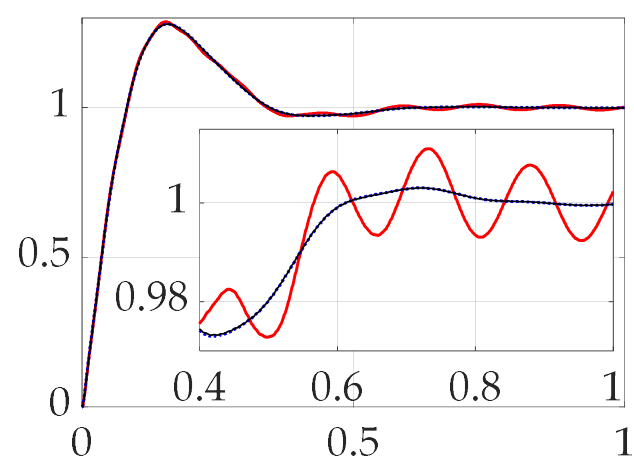

(a)
Table 2. Compensation of rigid body plus first two flexible modes (resonance and anti-resonance) with step input.

\begin{tabular}{|c|c|c|c|}
\hline System & $\begin{array}{c}\text { Gain } \\
\text { margin } \\
\text { (dB) }\end{array}$ & $\begin{array}{c}\text { Phase } \\
\text { Margin } \\
\text { (degrees) }\end{array}$ & $\begin{array}{c}\text { Stable/Un- } \\
\text { stable }\end{array}$ \\
\hline Uncontrolled & 64.5 & 0.000673 & Stable \\
\hline PID controlled & 4.95 & 17.9 & Stable \\
\hline PID + mode 1 & $-25.9^{1}$ & 133 & Stable \\
\hline PID + mode $1+$ mode 2 & 5.52 & 18.3 & Stable \\
\hline $\begin{array}{c}\text { PID }+ \text { mode } 1+\text { mode } 2 \\
+ \text { bandpass }\end{array}$ & 0.625 & 4.01 & Stable \\
\hline
\end{tabular}

(b)

Figure 18. Five computer simulations of control of rigid body; compensated first flexible mode, compensated second anti-resonance, and fully compensated first two modes with frequency in hertz on the abscissa and response magnitude in degrees on the ordinate. (a) PID (thick, solid red line); PID + mode 1 + bandpass 2 (dotted blue line); PID + mode 1 + bandpass 2 + notch 2 (thin, solid black line) again illustrating substantial improvement by compensating for additional (second) anti-resonance, but negligible difference also compensating for first additional (second).

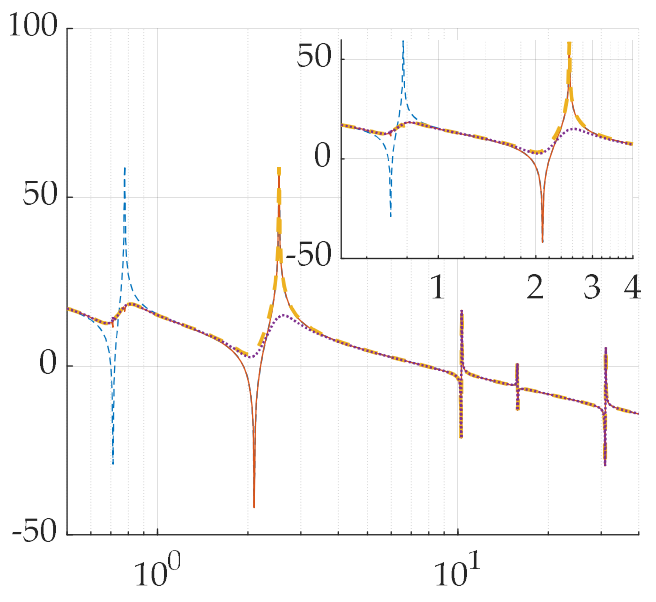

(a)

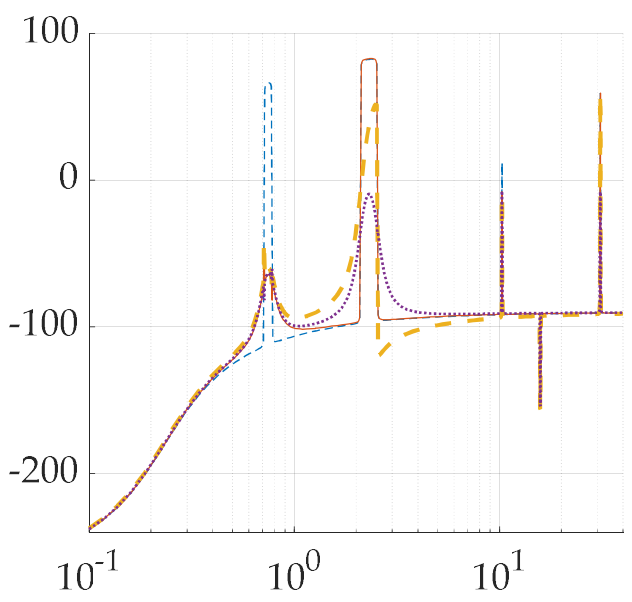

(b)

Figure 19. Four computer simulations of control of rigid body, first flexible mode and second flexible mode with frequency in hertz on the abscissa: uncontrolled (narrow, dashed line), PID compensated, filtered first mode (thin, solid line), PID compensated, filtered first mode, plus bandpass filter of second antiresonance (thick, dashed line), and PID compensated, filtered first mode and second mode - anti-resonance and resonance (dotted line) NOTE WATERBED EFFECT (a) Response magnitude (first mode zoomed in the inset) on the ordinate, (b) Phase in degrees on the ordinate. 


\subsection{Gain stabilization plus structural filters (bandpass $\mathcal{E}$ notch) for first three flexible modes}

Gain stabilization with fully compensated first mode was augmented with structural filters using equation (33) where structural resonant frequencies were used as filter natural frequencies and filter damping was adjusted to visually "flatten the curve" of the frequency response plot at the location of the second mode. First, a bandpass variant was designed for the second anti-resonant spike. Next, a notch variant was designed for the second resonant spike. Figure 20 displays the results. Notice once again in the step response plot in subfigure (a) the nominal performances are nearly indistinguishable, while in the "inset" plot distinct differences are apparent, where quantitative results are displayed in Table 3 inserted as subfigure (b). Figure 21 displays four frequency response plots overlayed. The nominal frequency response (after gain stabilization) is indicated by the dashed blue line. The thin-solid red line indicates addition of full compensation of the first resonant mode. Meanwhile the overlaid dashed gold line indicates bandpass compensation of the second anti-resonance. Finally, the "flattened" frequency response plot's thin, dotted black line indicates full mode compensation with the addition of a notch filter at the first three structural resonant frequencies. Effects on frequency response phase is displayed in subfigure (b). As with the case of compensating for the second structural modes, addition of both bandpass filter at the third anti-resonance and notch filter at the third resonance increases reduces both gain and phase margin substantially. Compensation of the third resonant structure mode should therefore be avoided, despite an instinct to "flatten the curve" of the frequency response plot magnitude.

\subsection{Gain stabilization plus structural filters (bandpass $\mathcal{E}$ notch) for first four flexible modes}

Gain stabilization with fully compensated first mode was augmented with structural filters using equation (33) where structural resonant frequencies were used as filter natural frequencies and filter damping was adjusted to visually "flatten the curve" of the frequency response plot at the location of the second mode. First, a bandpass variant was designed for the second anti-resonant spike. Next, a notch variant was designed for the second resonant spike. Figure 22 displays the results. Notice once again in the step response plot in subfigure (a) the nominal performances are nearly indistinguishable, while in the "inset" plot distinct differences are apparent, where quantitative results are displayed in Table 4 inserted as subfigure (b). Figure 23 displays four frequency response plots overlayed. The nominal frequency response (after gain stabilization) is indicated by the dashed blue line. The thin-solid red line indicates addition of full compensation of the first resonant mode. Meanwhile the overlaid dashed gold line indicates bandpass compensation of the second anti-resonance. Finally, the "flattened" frequency response plot's thin, dotted black line indicates full mode compensation with the addition of a notch filter at the first three structural resonant frequencies. Effects on frequency response phase is displayed in subfigure (b). Different than the cases of compensating for the second and third structural modes, addition of the bandpass filter at the fourth anti-resonance restores outstanding gain margin performance, but addition of the notch filter at the fourth resonance does not restore phase margin performance. Compensation of the fourth resonant structure mode does not exceed nominal performance compensating for only the first vibrational mode and should therefore be avoided, despite an instinct to "flatten the curve" of the frequency response plot magnitude. 


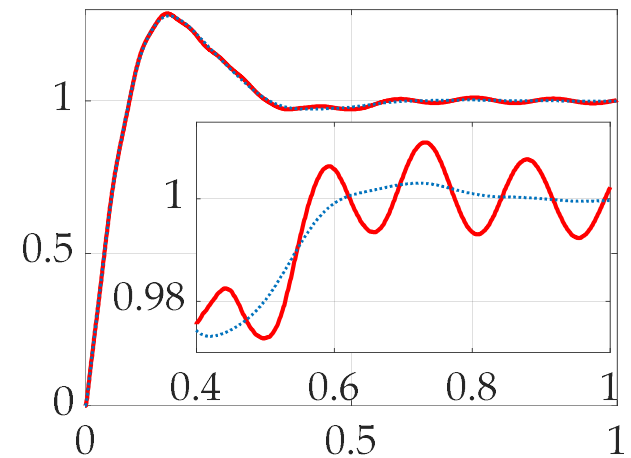

(a) PID (thick, solid red line); PID + modes 1-2 + bandpass 3 (dotted blue line); PID + modes 1-3 (thin, solid black line) again illustrating substantial improvement by compensating for additional (second) anti-resonance, but negligible difference also compensating for first additional (second).
Table 3. Compensation of rigid body plus first three flexible modes (resonance and anti-resonance) with step input.

\begin{tabular}{cccc}
\hline System & $\begin{array}{c}\text { Gain } \\
\text { margin } \\
\text { (dB) }\end{array}$ & $\begin{array}{c}\text { Phase } \\
\text { Margin } \\
\text { (degrees) }\end{array}$ & $\begin{array}{c}\text { Stable/Un- } \\
\text { stable }\end{array}$ \\
\hline Uncontrolled & 64.5 & 0.000673 & Stable \\
\hline PID controlled & 4.95 & 17.9 & Stable \\
\hline PID + mode 1 & $\mathbf{- 2 5 . 9 1}$ & $\mathbf{1 3 3}$ & Stable \\
\hline $\begin{array}{c}\text { PID + mode 2 + band- } \\
\text { pass }\end{array}$ & 3.87 & 16.4 & Stable \\
\hline PID + mode 2 + mode3 & 5.52 & 18.3 & Stable \\
\hline
\end{tabular}

${ }^{1}$ Negative gain margins indicate that stability is lost by decreasing the gain, while positive gain margins indicate that stability is lost by increasing the gain.

Figure 20. Five computer simulations of control of rigid body; compensated first flexible mode, compensated second anti-resonance, and fully compensated first two modes with frequency in hertz on the abscissa and response magnitude in degrees on the ordinate.

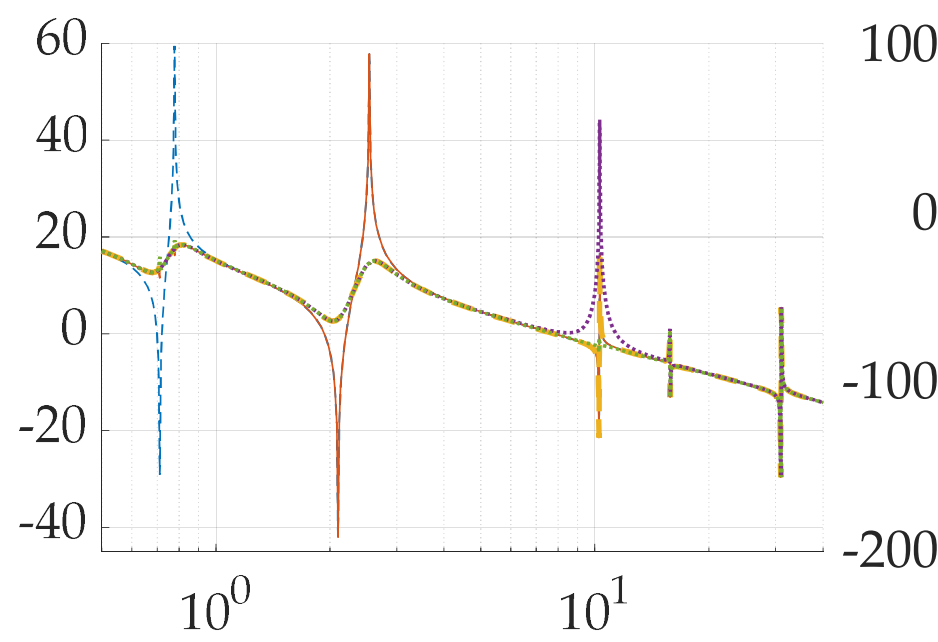

(a)

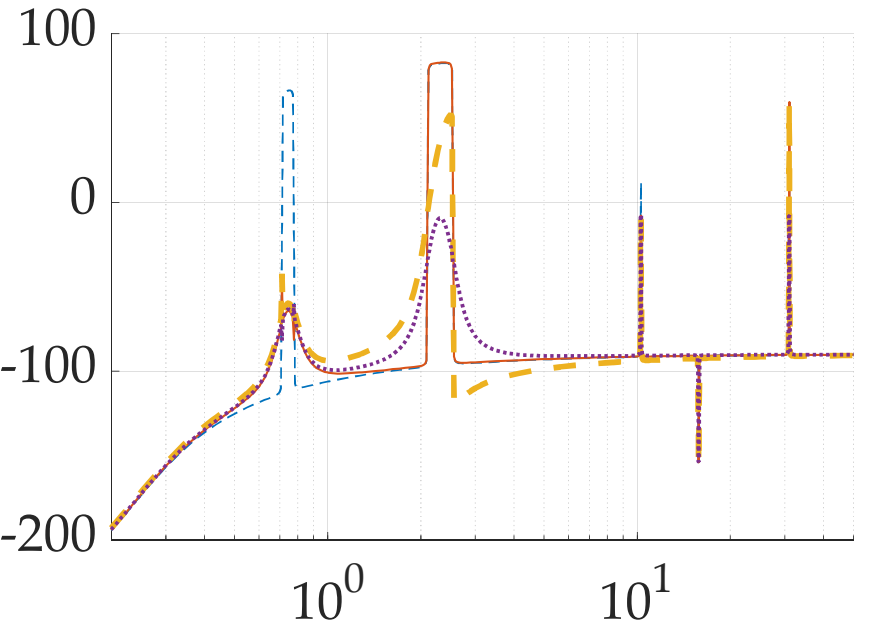

(b)

Figure 21. Six computer simulations of control of rigid body, first flexible mode, second flexible mode, and third flexible mode with frequency in hertz on the abscissa: uncontrolled (narrow, dashed line), PID compensated, filtered first mode (thin, solid line), PID compensated, filtered first mode, PID compensated, filtered first and second mode (thick, dashed line), and PID compensated, filtered first, second, and third mode - anti-resonance and resonance (dotted line) NOTE WATERBED EFFECT (a) Response magnitude (first mode zoomed in the inset) on the ordinate, (b) Phase in degrees on the ordinate. DOUBLE CHECK 


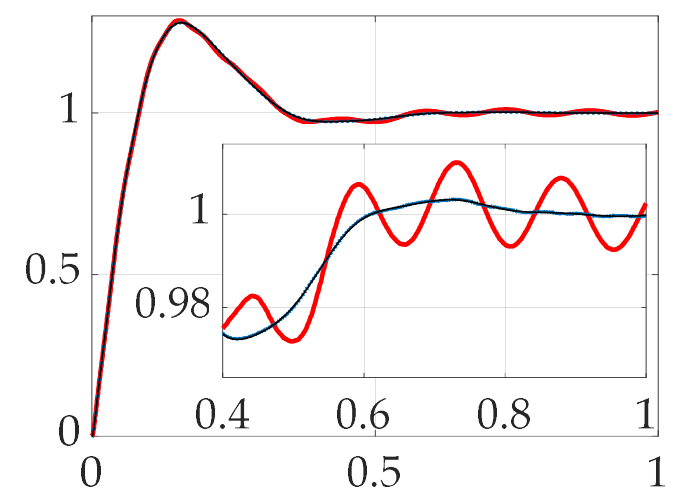

(a) PID (thick, solid red line); PID + modes 1$3+$ bandpass 4 (dotted blue line); PID + modes 1-4 (thin, solid black line) again illustrating substantial improvement by compensating for additional (second) anti-resonance, but negligible difference also compensating for first additional (second).
Table 4. Compensation of rigid body plus four flexible modes (resonance and anti-resonance) with step input.

\begin{tabular}{cccc}
\hline System & $\begin{array}{c}\text { Gain } \\
\text { margin } \\
\text { (dB) }\end{array}$ & $\begin{array}{c}\text { Phase } \\
\text { Margin } \\
\text { (degrees) }\end{array}$ & $\begin{array}{c}\text { Stable/Un- } \\
\text { stable }\end{array}$ \\
\hline Uncontrolled & 64.5 & 0.000673 & Stable \\
\hline PID controlled & 4.95 & 17.9 & Stable \\
\hline PID + mode 1 & -25.91 & $\mathbf{1 3 3}$ & Stable \\
\hline PID + mode 1 - 2 & 5.52 & 18.3 & Stable \\
\hline PID + mode 1 - 3 & 5.89 & 18.4 & Stable \\
\hline $\begin{array}{c}\text { PID + mode 1 - 3 } \\
\text { bandpass 4 }\end{array}$ & $\mathbf{- 2 6 . 8}$ & 17 & Stable \\
\hline PID + mode 1 - 4 & 6.1 & 18.5 & Stable \\
\hline
\end{tabular}

${ }^{1}$ Negative gain margins indicate that stability is lost by decreasing the gain, while positive gain margins indicate that stability is lost by increasing the gain.

Figure 22. Five computer simulations of control of rigid body and fully compensated modes with frequency in hertz on the abscissa and response magnitude in degrees on the ordinate.

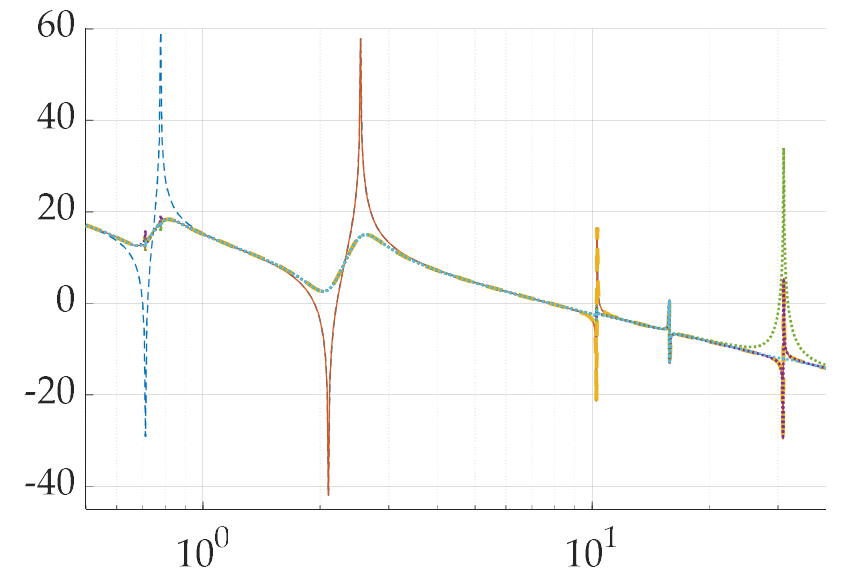

(a)

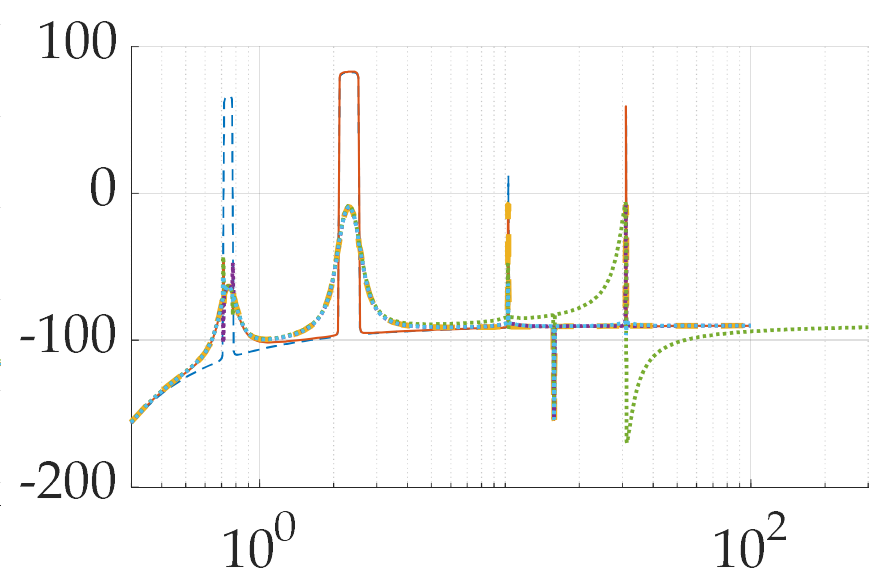

(b)

Figure 23. Six computer simulations of control of rigid body, first flexible mode, second flexible mode, third flexible mode and fourth flexible mode with frequency in hertz on the abscissa: uncontrolled (narrow, dashed line), PID compensated, filtered first mode (thin, solid line), PID compensated, filtered first mode, PID compensated, filtered first and second mode (thick, dashed line), and PID compensated, filtered first, second, and third mode - anti-resonance and resonance (dotted line) NOTE WATERBED EFFECT (a) Response magnitude (first mode zoomed in the inset) on the ordinate, (b) Phase in degrees on the ordinate. DOUBLE CHECK 
3.6 Comparisons of stability margins: Gain stabilization plus structural filters (bandpass $\mathcal{E}$
notch)

This section summarizes the incrementally achieved results thus far in table 5. Noteworthy performances are highlighted in bold font indicating compensation of the first mode alone following gain compensation is the superior method based on a combined measure of both gain and phase margin. Meanwhile, additional compensation of the first three structural modes plus the fourth anti-resonance produce the highest gain margin but suffer from reduction in phase margin.

Table 5. Classical compensation of rigid body plus first four flexible modes (resonances and anti-resonances) with step inputs.

\begin{tabular}{ccc}
\hline System & $\begin{array}{c}\text { Percent improved } \\
\text { gain margin }\end{array}$ & $\begin{array}{c}\text { Percent improved } \\
\text { phase margin }\end{array}$ \\
\hline PID controlled & - & -- \\
\hline PID + mode 1 & $\mathbf{4 2 3 . 4 \%}$ & $\mathbf{6 4 3 . 0 \%}$ \\
\hline PID + mode 1 + mode 2 & $11.5 \%$ & $2.2 \%$ \\
\hline PID + mode 1 + mode 2 + mode 3 & $19.0 \%$ & $2.8 \%$ \\
\hline PID + mode 1 + mode 2 + mode 3 + bandpass & $\mathbf{4 4 1 . 4 \%} \%$ & $-5.0 \%$ \\
\hline PID + mode 1 + mode 2 + mode 3 + mode 4 & $23.2 \%$ & $3.4 \%$ \\
\hline \multirow{2}{*}{ 1 Negative gain margins indicate that stability is lost by decreasing the gain, while positive gain } \\
margins indicate that stability is lost by increasing the gain.
\end{tabular}

From these results comes the practice of not fully compensating for all flexible modes in favor of compensating only for the first flexible mode in addition to benchmark gain stabilization. Next consider utilizing single sinusoidally shaped step inputs to improve target tracking performance.

\subsection{Sinusoidal trajectory shaping: Gain stabilization plus structural filters of only selected modes}

Assuming the best performing compensator identified in section 3.6 (benchmark gain stabilization with classical compensation of the first flexile mode's anti-resonance and resonance), this section addresses shaping the step command to further increase performance, where the results are depicted in figure 24 comparing step trajectories versus single-sinusoidal shaped-trajectories mimicking step functions. The figure of merits used for comparison include gain margin and phase margin (listed in table 5), and a trajectory tracking error in table 6 with percentage performance improvement.

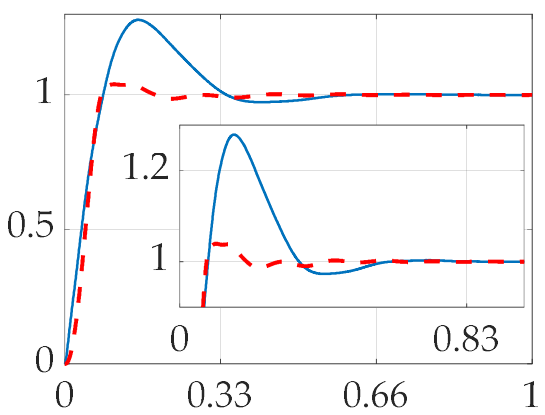

(a) Step responses

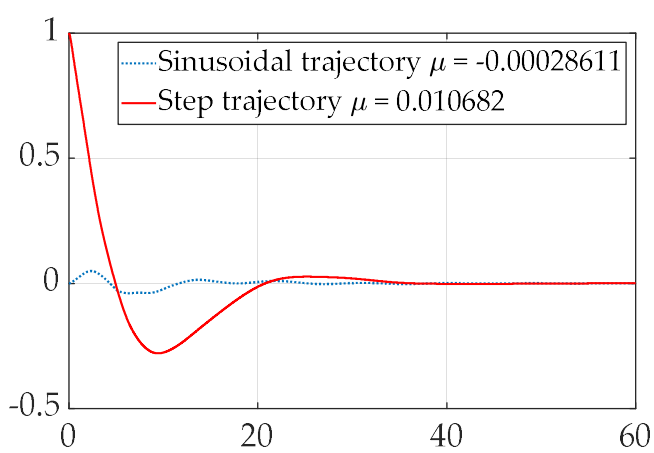

(b) Error (degrees)

Figure 24. Utilizing sinusoidal-shaped trajectories to improve step response. (a) responses for step and sinusoidal trajectories, and (b) tracking error following sinusoidal and step trajectories.

Notice sinusoidal trajectory shaping is compared to two other modern benchmarks in figure 25: shaped-whiplash and time-delay input shaped. The latter two methods do not improve performance when combined with classical feedback compensation, which seems logical since the methods are derived to act alone. Meanwhile trajectory shaping with 
single-frequency sinusoids with simultaneous classical mode compensation improve performance two orders of magnitude.

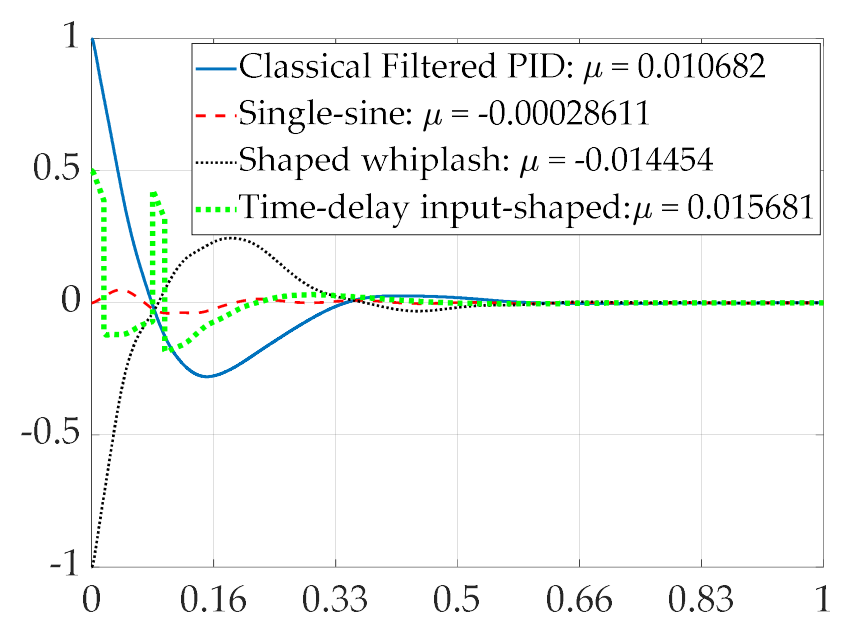

(a)
Table 6. Controlling rigid body with four flexible modes (resonance and anti-resonance) with step inputs.

\begin{tabular}{ccc}
\hline Shaping method & $\begin{array}{c}\text { Mean } \\
\text { tracking } \\
\text { error } \\
\text { (deviation) }\end{array}$ & $\begin{array}{c}\text { Percent } \\
\text { performance } \\
\text { improvement }\end{array}$ \\
\hline No trajectory shaping & 0.010682 & -- \\
Single sine shaping & $\mathbf{0 . 0 0 0 2 8 6 1 1}$ & $\mathbf{9 7}$ \\
Shaped whiplash & 0.014454 & 35 \\
Time-delayed shaping & 0.015681 & 47 \\
\hline
\end{tabular}

(b)

Figure 25. Comparing whiplash compensation, time-delay shaping, filtered PID (with step trajecotries) and single-sinusoidal trajectories. (a) error in degrees on the ordinate with displacement on the abscissa; (b) table of mean tracking errors and performance improvement percentages

Section 3 includes simulation results presented in both qualitative fashion vis multiplots, but also quantitatively using ubiquitous figures of merit: gain margin, phase margin, mean error, and finally percent performance improvement. Conclusions include:

1. Superior performance (based on gain margin, phase margin, and mean tracking error) is attained by only compensating for the first complete flexible mode (resonance and anti-resonance) with notch and bandpass filters respectively with step commands shaped by novel single-sinusoidal trajectories.

a. The next-best performer was novel shaped-whiplash control based on [23].

2. Specific compensation of higher modes did not substantially improve performance with the exception of fully compensating the rigid body, first three flexible modes and bandpass filter the fourth anti-resonance. This option very substantially improved gain margin, while slightly decreasing phase margin.

The results are section three are summarized in general terms in the discussion of section 4, emphasizing percent performance improvement over the declared benchmark.

\section{Discussion}

Instincts to improve system performance by addressing maladies are tempered by a curse of dimensionality. As more and more maladies are addressed, system order (of the governing differential equations) increases and so does complication in coupled systems of differential equations. This notion manifests in two regards: stability and trajectory tracking performance. In terms of stability, the system is enhanced most by compensating for the first flexible mode alone with deleterious effects resulting from additional compensation of higher vibrational modes. On the other hand, target tracking performance increases with system order. Augmentation of nominal step inputs (table 7) with sinusoidal shaped trajectories (table 8) leads to substantially larger improvements than using step functions and furthermore achieves best performance at relatively lower system order.

\subsection{Comparison(s) summary: mean errors and percent performance improvements}

Table 7 indicates classical mode compensation increases performance over twenty percent, while addition of sinusoidally shaped trajectories raises the performance increase to 
well over ninety percent at lower system orders as displayed in table 8. Best results are achieved by compensating for the first complete flexible mode (both resonance and antiresonance) with bandpass and notch filters respectively and shaping commanded trajectories with single-frequency sinusoids. Over ninety-eight percent performance improvement is achieved compared to classical PID control with step inputs.

RECOMMENDATION: use single frequency, sinusoidally shaped step functions as inputs to flexible space robots and compensate for flexible modes classically as preferred.

Table 7. Mean trajectory tracking error of step responses.

\begin{tabular}{cccc}
\hline System with step input & System order & Mean error & Percent improvement \\
\hline PID controlled with step input (reference) & 26 & 0.013984 & - - \\
\hline PID + mode 1 & 34 & 0.013848 & 0.97 \\
\hline PID + mode 1 + mode 2 & 42 & 0.010795 & 22.8 \\
\hline PID + mode 1 + mode 2 + mode 3 & $\mathbf{5 0}$ & $\mathbf{0 . 0 1 0 6 8 2}$ & $\mathbf{2 3 . 6}$ \\
\hline
\end{tabular}

Table 8. Mean tracking errors using step responses with sinusoidal-shaped trajectories.

\begin{tabular}{cccc}
\hline System with single-sinusoidal shaped trajectory & System order & Mean error & Percent improvement \\
\hline PID controlled with step input (benchmark) & 26 & 0.013984 & - - \\
\hline PID controlled & 26 & 0.000502 & 96.4 \\
\hline PID + mode 1 & 34 & $\mathbf{0 . 0 0 0 1 9 9}$ & $\mathbf{9 8 . 6}$ \\
\hline PID + mode 1 + mode 2 & 42 & 0.00021 & 98.4 \\
\hline PID + mode 1 + mode 2 + mode 3 & 50 & -0.00029 & 98.0 \\
\hline
\end{tabular}

\subsection{Future research recommendations}

Particularly in light of the superior performance of single-sine shaped input trajectories, deterministic artificial intelligence is a natural recommendation for sequel research, especially since the method's current instantiation emphasizes single-sine trajectory shaping. The combination of deterministic artificial intelligence will leverage the work presented in this manuscript to track time-varying natural vibration modes as a matter of fact of the two methods' shared formulation.

Funding: Please add: This research received no external funding. The APC was funded by the author.

Data Availability Statement: Data supporting reported results can be obtained by contacting the author.

Conflicts of Interest: The author declares no conflict of interest.

\section{References}

1. Kremer, K. Robotics Refueling Research Scores Huge Leap at Space Station. The Universe Today: Space and Astronomy News. March 24, 2012. Available online, accessed 15 January 2022: https://www.universetoday.com/94122/robotics-refueling-researchscores-huge-leap-at-space-station/

2. Naval Postgraduate School, Spacecraft Robotics Laboratory. Accessible online, accessed 15 January 2022 : https://nps.edu/web/srl

3. RRM Task: Refueling - NASA's Exploration \& In-space Services. Available online, accessed January 16, 2022: https://nexis.gsfc.nasa.gov/rrm_refueling_task.html

4. Marshall Spaceflight Center, Advanced Space Transportation Program: Paving the Highway to Space. Accessible online, accessed 15 January 2022: https:/www.nasa.gov/centers/marshall/news/background/facts/astp.html

5. Maxar and NASA will demonstrate orbital spacecraft assembly with a new robotic arm. Available online, accessed January 16, 2022 at: https:/techcrunch.com/2020/01/31/maxar-and-nasa-will-demonstrate-orbital-spacecraft-assembly-with-a-new-roboticarm/

6. Spacecraft Research \& Design Center. Available online, accessed January 16, 2022: https://nps.edu/web/srdc/laboratories 
7. Chasles, M. Note sur les propriétés générales du système de deux corps semblables entr'eux. Bull. Sci. Math. Astron. Phys. Chem. 1830, 14, 321-326. (In French)

8. Newton, I. Principia, Jussu Societatis Regix ac Typis Joseph Streater; Cambridge University Library: London, UK, 1687.

9. Euler, L. Formulae Generales pro Translatione Quacunque Corporum Rigidorum (General Formulas for the Translation of Arbitrary Rigid Bodies. Novi Comment. Acad. Sci. Petropolitanae 1776, 20, 189-207. Available online: https://math.dartmouth.edu/ euler/docs/originals/E478.pdf (accessed on 2 November 2019).

10. Wie, B. Space Vehicle Dynamics and Control. 2nd, American Institute of Aeronautics and Astronautics, Reston, VA, 2008.

11. Agrawal, B., Design of Geosynchronous Spacecraft, Prentice-Hall: New Jersey USA, 1986, p. 204.

12. Likens, P.; Dynamics and Control of Flexible Space Vehicles, NASA Technical Report 32-1329, February 15, 1969.

13. Bellman, R.E. Dynamic Programming; Princeton University Press: Princeton, NJ, USA, 1957; ISBN 0-486-42809-5

14. Kwakernaak, H., Sivan, R. (1972). Linear Optimal Control Systems. First Edition. Wiley-Interscience. ISBN 0-471-51110-2.

15. Maryamnegari, M.; Khoshnood, H; Robust adaptive vibration control of an underactuated flexible spacecraft. J. Vib.Con. 2019 25(4), 834-850. doi:10.1177/1077546318802431

16. Abad, F.; Zheng, W.; Ou, M.; Khanh, P. Optimal Control of Space Robots for Capturing a Tumbling Object with Uncertainties. J. Guid. Con. Dyn.2014, 37. 1-4. doi: 10.2514/1.G000003.

17. Singhose, W.; Seering, W.; Singer, M. Input Shaping for Vibration Reduction with Specified Insensitivity to Modeling Errors. In Proceedings of Japan-USA Symposium on Flexible Automation, 1. Boston, Massachusetts, USA. July 7-10, 1996.

18. Pao, L. Multi-input shaping design for vibration reduction, Automatica 1999 35, 1, 81-89 doi: 10.1016/S0005-1098(98)00124-1.

19. Gorinevsky, D., Vukovich, G.; Nonlinear Input Shaping Control of Flexible Spacecraft Reorientation Maneuver. J. Guid. Con. Dyn 1998 21(2), 264-270.

20. K., Zhengxian, Y. Combined feedback control and input shaping for vibration suppression of flexible spacecraft. In proceedings of the International Conference on Mechatronics and Automation, pp. 3257-3262, Changchun, China. 18 September 2009 doi: 10.1109/ICMA.2009.5246238.

21. Audin, M. Hamiltonian systems and their integrability. American Mathematical Society: Providence, R.I., USA 2008; ISBN 978-08218-4413-7

22. Pontryagin, L.; Boltyanskii, V.; Gamkrelidze, R.; Mischenko, E. The Mathematical Theory of Optimal Processes; Neustadt, L.W., Ed.; Wiley: New York, NY, USA, 1962.

23. Sands, T. Optimization Provenance of Whiplash Compensation for Flexible Space Robotics. Aerospace 2019, 6(9), 93. doi: 10.3390/aerospace6090093.

24. Sands, T. Development of deterministic artificial intelligence for unmanned underwater vehicles (UUV). J. Mar. Sci. Eng. 2020, 8(8),578. doi: 10.3390/jmse8080578.

25. Baker, K.; Cooper, M.; Heidlauf, P.; Sands, T. Autonomous trajectory generation for deterministic artificial intelligence. Electr. Electron. Eng. 2018, 8(3), 59. doi:10.5923/j.eee.20180803.01

26. Sands, T.; Kim, J.; Agrawal, B. Spacecraft Adaptive Control Evaluation. In Proceedings of the Infotech@ Aerospace, Garden Grove, CA, USA, 19-21 June 2012; American Institute of Aeronautics and Astronautics: Reston, VA, USA, 2012; pp. 2012-2476.

27. Smeresky, B.; Rizzo, A.; Sands, T. Optimal Learning and Self-Awareness Versus PDI. Algorithms 2020, 13, 23. doi: 10.3390/a13010023

28. Badrieh F. Properties of the Fourier Transforms. In: Spectral, Convolution and Numerical Techniques in Circuit Theory. Springer International Publishing AG, part of Springer Nature 2018. doi: 10.1007/978-3-319-71437-0_9

29. Physics Forums, 2022, Fourier of a unit step signal. https://www.physicsforums.com/threads/fourier-of-unit-step-signal.620990/ Available online: (accessed 13 June 13, 2021)

30. Hamilton, W. On a General Method in Dynamics; Royal Society: London, UK, 1834; pp. 247-308.

31. Coriolis, G. (1835). "Sur les équations du mouvement relatif des systèmes de corps". Journal de l'École Royale Polytechnique. 1835 15, 144-154.

32. Merz, J. A History of European Thought in the Nineteenth Century; Blackwood: London, UK, 1903; p. 5.

33. Whittaker, E. A Treatise on the Analytical Dynamics of Particles and Rigid Bodies; Cambridge University Press: New York, NY, USA, 1904; p. 1937.

34. Church, I. Mechanics of Engineering; Wiley: New York, NY, USA, 1908.

35. Wright, T. Elements of Mechanics Including Kinematics, Kinetics, and Statics, with Applications; Nostrand: New York, NY, USA, 1909.

36. Gray, A. A Treatise on Gyrostatics and Rotational Motion; MacMillan: London, UK, 1918; ISBN 978-1-4212-5592-7.

37. Rose, M. Elementary Theory of Angular Momentum; John Wiley \& Sons: New York, NY, USA, 1957; ISBN 978-0-486-68480-2.

38. Sands, T. Comparison and Interpretation Methods for Predictive Control of Mechanics. Algorithms 2019, 12, 232. doi: 10.3390/a12110232

39. Greenwood, D. Principles of Dynamics; Prentice-Hall: Englewood Cliffs, NJ, USA, 1965; ISBN 9780137089741.

40. Goldstein, H. Classical Mechanics, 2nd ed.; Addison-Wesley: Boston, MA, USA, 1981.

41. Kane, T. Analytical Elements of Mechanics Volume 1; Academic Press: New York, NY, USA; London, UK, 1959.

42. Kane, T. Analytical Elements of Mechanics Volume 2 Dynamics; Academic Press: New York, NY, USA; London, UK, 1961.

43. Kane, T.; Levinson, D. Dynamics: Theory and Application; McGraw-Hill: New York, NY, USA, 1985.

44. Roithmayr, C.; Hodges, D. Dynamics: Theory and Application of Kane's Method; Cambridge: New York, NY, USA, 2016. 
45. Chen, H.; Agrawal, B. Method of Slewing the Spacecraft to Minimize Settling Time. In proceedings of the AIAA Guidance, Navigation, and Control Conference and Exhibit, 5-8 August 2002, Monterey, California. Abstract Number AIAA $2002-4656$. doi: $10.2514 / 6.2002-4656$

46. Piezo Motion Control Tutorial - Piezo Basics for Precision Motion, Force Generation \& Nanopositioning Applications in Industry and Research. Available online, accessed on January 16, 2022: https://www.pi-usa.us/en/products/piezo-flexure-nanopositioners/piezo-motion-control-tutorial

47. Meyer, J.; Harrington, W.; Agrawal, B.; Song, G. Application of piezoceramics to vibration suppression of a spacecraft flexible appendage. In proceedings of the AIAA, Guidance, Navigation and Control Conference, San Diego, CA, July 29-31, 1996. Available online, accessed 26 Jan 2022 at: https://calhoun.nps.edu/bitstream/handle/10945/36769/Meyer_harrington_agrawal_song_Application_of_Piezo_1996.pdf?sequence=1\&isAllowed=y

48. Song, G.; Agrawal, B. Vibration suppression of flexible spacecraft during attitude control. Acta Astronautica 2001, 49(2), 73-83. doi: 10.1016/S0094-5765(00)00163-6

49. Breon, S.; Boyle, R.; Francom; M.; DeLee, C.; Francis, J.; Mustafi, S.; Barfknecht, P.; McGuire, J.; Krenn, A.; Zimmerli, G.; Hauser, D. Robotic Refueling Mission-3 - an overview. In Proceedings of the Cryogenic Engineering Conference (CEC), 21-25 July 2019, Hartford, Connecticut, USA. Document ID: 20190027535. Available online, accessed January 16, 2022: https://ntrs.nasa.gov/citations/20190027535

50. Mpanza, L.; Pedro, J. Optimised Tuning of a PID-Based Flight Controller for a Medium-Scale Rotorcraft. Algorithms 2021, 14, 178. https://doi.org/10.3390/a14060178

51. Zhao, Z.; Liu, S.; Pan, J. A PID Parameter Tuning Method Based on the Improved QUATRE Algorithm. Algorithms 2021, 14, 173. https://doi.org/10.3390/a14060173

52. Remoaldo, D.; Jesus, I. Analysis of a Traditional and a Fuzzy Logic Enhanced Perturb and Observe Algorithm for the MPPT of a Photovoltaic System. Algorithms 2021, 14, 24. https://doi.org/10.3390/a14010024

53. Borase, R.P., Maghade, D.K., Sondkar, S.Y. et al. A review of PID control, tuning methods and applications. Int. J. Dynam. Control 2021 9, 818-827. https://doi.org/10.1007/s40435-020-00665-4

54. Kim, K.; Moon, S.; Kim, J.; Park, Y.; Lee, J.-H. Input Shaping Based on an Experimental Transfer Function for an Electrostatic Microscanner in a Quasistatic Mode. Micromachines 2019, 10, 217. https://doi.org/10.3390/mi10040217

55. Dwi Astuti, W.; Matsukuma, H.; Nakao, M.; Li, K.; Shimizu, Y.; Gao, W. An Optical Frequency Domain Angle Measurement Method Based on Second Harmonic Generation. Sensors 2021, 21, 670. https://doi.org/10.3390/s21020670

56. Peláez, G.; Vaugan, J.; Izquierdo, P.; Rubio, H.; García-Prada, J.C. Dynamics and Embedded Internet of Things Input Shaping Control for Overhead Cranes Transporting Multibody Payloads. Sensors 2018, 18, 1817. https://doi.org/10.3390/s18061817

57. Singh, T.; Singhose, W. Tutorial on input shaping/time delay control of maneuvering flexible structures. In Proceedings of the American Control Conference, 3, 1717 - 1731. Anchorage, AK, USA May 8-10, 2002; doi: 10.1109/ACC.2002.1023813.

58. Input Shaping. Available online, accessed January 17, 2022 at :https://en.wikipedia.org/wiki/Input_shaping

59. Rush D. Robinett; Rush D. Robinett III; John Feddema; G. Richard Eisler; Clark Dohrmann; Gordon G. Parker; David G. Wilson; Dennis Stokes (2001). Flexible Robot Dynamics and Controls. Kluwer Academic/Plenum (Springer), New York USA. ISBN 0-30646724-0. doi: 10.1007/978-1-4615-0539-6

60. Sands, T. Virtual Sensoring of Motion Using Pontryagin's Treatment of Hamiltonian Systems. Sensors 2021, 21, 4603. https://doi.org/10.3390/s21134603 


\section{Appendix A Elaboration of modal system identification on the flexible robot system}

Substitute into equation (19):

$$
\begin{gathered}
\ddot{\theta}+\frac{\sum_{i=1}^{n} D_{i}\left(-2 \xi \omega_{i} I_{z z}\right)}{I_{z z}\left(I_{z z}-D_{i} \sum_{i=1}^{n} D_{i}\right)} \dot{q}_{i}+\frac{\sum_{i=1}^{n} D_{i}\left(\omega_{i}^{2} I_{z z}\right)}{I_{z z}\left(I_{z z}-D_{i} \sum_{i=1}^{n} D_{i}\right)} q_{i}+\frac{\sum_{i=1}^{n} D_{i}\left(-D_{i} T\right)}{I_{z z}\left(I_{z z}-D_{i} \sum_{i=1}^{n} D_{i}\right)}=\frac{T}{I_{z z}} \\
\ddot{\theta}=\frac{2 \xi \omega_{i} \sum_{i=1}^{n} D_{i}}{I_{z z}-D_{i} \sum_{i=1}^{n} D_{i}} \dot{q}_{i}+\frac{\omega_{i}^{2} \sum_{i=1}^{n} D_{i}}{I_{z z}-D_{i} \sum_{i=1}^{n} D_{i}} q_{i}+\frac{T D_{i} \sum_{i=1}^{n} D_{i}}{I_{z z}\left(I_{z z}-D_{i} \sum_{i=1}^{n} D_{i}\right)}=\frac{T}{I_{z z}} \\
\ddot{\theta}=\frac{2 \xi \omega_{i} \sum_{i=1}^{n} D_{i}}{I_{z z}-D_{i} \sum_{i=1}^{n} D_{i}} \dot{q}_{i}+\frac{\omega_{i}^{2} \sum_{i=1}^{n} D_{i}}{I_{z z}-D_{i} \sum_{i=1}^{n} D_{i}} q_{i}+\frac{T\left(D_{i} \sum_{i=1}^{n} D_{i}+I_{z z}-D_{i} \sum_{i=1}^{n} D_{i}\right)}{I_{z z}\left(I_{z z}-D_{i} \sum_{i=1}^{n} D_{i}\right)} \\
\ddot{\theta}=\frac{2 \xi \omega_{i} \sum_{i=1}^{n} D_{i}}{I_{z z}-D_{i} \sum_{i=1}^{n} D_{i}} \dot{q}_{i}+\frac{\omega_{i}^{2} \sum_{i=1}^{n} D_{i}}{I_{z z}-D_{i} \sum_{i=1}^{n} D_{i}} q_{i}+\frac{T I_{z z}}{I_{z z}\left(I_{z z}-D_{i} \sum_{i=1}^{n} D_{i}\right)} \\
\ddot{\theta}=\frac{2 \xi \omega_{i} \sum_{i=1}^{n} D_{i}}{I_{z z}-D_{i} \sum_{i=1}^{n} D_{i}} \dot{q}_{i}+\frac{\omega_{i}^{2} \sum_{i=1}^{n} D_{i}}{I_{z z}-D_{i} \sum_{i=1}^{n} D_{i}} q_{i}+\frac{T}{\left(I_{z z}-D_{i} \sum_{i=1}^{n} D_{i}\right)}
\end{gathered}
$$

Recall the expressions for the rigid elastic coupling using modal coordinates: $D_{i}=\int_{F}\left(x_{F} \phi_{i}^{y}-y_{F} \phi_{i}^{x}\right) d m$ where

\begin{tabular}{|c|c|c|c|c|c|c|c|c|c|c|c|c|c|c|}
\hline & W2 & $\theta 2$ & W3 & $\theta 3$ & W4 & $\theta 4$ & W5 & $\theta 5$ & U6 & $\theta 6$ & u7 & $\theta 7$ & u8 & $\theta 8$ \\
\hline W2 & 958.8179 & 0.0000 & -479.409 & 59.9261 & 0 & 0 & 0 & 0 & 0 & 0 & 0 & 0 & 0 & 0 \\
\hline$\theta 2$ & 0.0000 & 19.9754 & -59.9261 & 4.9938 & 0 & 0 & 0 & 0 & 0 & 0 & 0 & 0 & 0 & 0 \\
\hline W3 & -479.409 & -59.926 & 958.8179 & 0.0000 & -479.409 & 59.9261 & 0 & 0 & 0 & 0 & 0 & 0 & 0 & 0 \\
\hline$\theta 3$ & 59.9261 & 4.9938 & 0.0000 & 19.9754 & -59.9261 & 4.9938 & 0 & 0 & 0 & 0 & 0 & 0 & 0 & 0 \\
\hline W4 & 0 & 0 & -479.409 & -59.926 & 958.8179 & 0.0000 & -479.409 & 59.9261 & 0 & 0 & 0 & 0 & 0 & 0 \\
\hline$\theta 4$ & 0 & 0 & 59.9261 & 4.9938 & 0.0000 & 19.9754 & -59.9261 & 4.9938 & 0 & 0 & 0 & 0 & 0 & 0 \\
\hline W5 & 0 & 0 & 0 & 0 & -479.409 & -59.926 & 479.409 & -59.926 & 0 & 0 & 0 & 0 & 0 & 0 \\
\hline$\theta 5$ & 0 & 0 & 0 & 0 & 59.9261 & 4.9938 & -59.9261 & 19.9754 & -59.9261 & 4.9938 & 0 & 0 & 0 & 0 \\
\hline U6 & 0 & 0 & 0 & 0 & 0 & 0 & 0 & -59.926 & 958.8179 & 0.0000 & -479.409 & 59.9261 & 0 & 0 \\
\hline$\theta 6$ & 0 & 0 & 0 & 0 & 0 & 0 & 0 & 4.9938 & 0.0000 & 19.9754 & -59.9261 & 4.9938 & 0 & 0 \\
\hline U7 & 0 & 0 & 0 & 0 & 0 & 0 & 0 & 0 & -479.409 & -59.926 & 958.8179 & 0.0000 & -479.409 & 59.9261 \\
\hline$\theta 7$ & 0 & 0 & 0 & 0 & 0 & 0 & 0 & 0 & 59.9261 & 4.9938 & 0.0000 & 19.9754 & -59.9261 & 4.9938 \\
\hline U8 & 0 & 0 & 0 & 0 & 0 & 0 & 0 & 0 & 0 & 0 & -479.409 & -59.926 & 479.4089 & -59.926 \\
\hline$\theta 8$ & 0 & 0 & 0 & 0 & 0 & 0 & 0 & 0 & 0 & 0 & 59.9261 & 4.9938 & -59.9261 & 9.9877 \\
\hline
\end{tabular}
$\phi$ 's are mode shapes from finite element analysis using the eigenvalues of $\mathrm{K} / \mathrm{m}$ (stiffness/mass). The system stiffness matrix is included in table A1 and mass matrix in table A2 result in the natural frequencies and mode shapes for the flexible system in table A3 and A4.

Table A1. Stiffness matrix [K]

${ }^{1}$ Notice state sequence alternates translation, then rotation at each node

Table A3. Natural frequencies, $\omega_{n}[\mathrm{rad} / \mathrm{s}]$

\begin{tabular}{ccccc}
\hline 1809.5 & 1415.5 & 1042.2 & 774.3 & 596.8 \\
478.8 & 410.9 & 54.9 & 43.7 & 30.9 \\
15.8 & 10.2 & 0.7 & 2.1 & \\
\hline \multicolumn{7}{c}{}
\end{tabular}

${ }^{1}$ corresponding to mode shapes in table 5 . 
Table A2. Mass matrix [M]

\begin{tabular}{|c|c|c|c|c|c|c|c|c|c|c|c|c|c|c|}
\hline Mass & W2 & $\theta 2$ & W3 & $\theta 3$ & W4 & $\theta 4$ & W5 & $\theta 5$ & U6 & $\theta 6$ & $\mathrm{u} 7$ & $\theta 7$ & u8 & $\theta 8$ \\
\hline W2 & 0.4761 & 0.0000 & 0.0037 & -0.0002 & 0 & 0 & 0 & 0 & 0 & 0 & 0 & 0 & 0 & 0 \\
\hline$\theta 2$ & 0.0000 & 0.0000 & 0.0002 & -0.0001 & 0 & 0 & 0 & 0 & 0 & 0 & 0 & 0 & 0 & 0 \\
\hline W3 & 0.0037 & 0.0002 & $4.76 \mathrm{E}-01$ & 0.0000 & 0.0037 & -0.0002 & 0 & 0 & 0 & 0 & 0 & 0 & 0 & 0 \\
\hline$\theta 3$ & -0.0002 & -0.0001 & 0.0000 & 0.0000 & 0.0002 & -0.0001 & 0 & 0 & 0 & 0 & 0 & 0 & 0 & 0 \\
\hline W4 & 0 & 0 & 0.0037 & 0.0002 & 0.4761 & 0.0000 & 0.0037 & -0.0002 & 0 & 0 & 0 & 0 & 0 & 0 \\
\hline$\theta 4$ & 0 & 0 & -0.0002 & -0.0001 & 0.0000 & 0.0000 & 0.0002 & -0.0001 & 0 & 0 & 0 & 0 & 0 & 0 \\
\hline W5 & 0 & 0 & 0 & 0 & 0.0037 & 0.0002 & $2.63 \mathrm{E}+00$ & -0.0004 & 0 & 0 & 0 & 0 & 0 & 0 \\
\hline$\theta 5$ & 0 & 0 & 0 & 0 & -0.0002 & -0.0001 & -0.0004 & 0.0000 & 0.0002 & -0.0001 & 0 & 0 & 0 & 0 \\
\hline U6 & 0 & 0 & 0 & 0 & 0 & 0 & 0 & 0.0002 & 4.76E-01 & 0.0000 & 0.0037 & -0.0002 & 0 & 0 \\
\hline$\theta 6$ & 0 & 0 & 0 & 0 & 0 & 0 & 0 & -0.0001 & $0.00 \mathrm{E}+00$ & 0.0000 & 0.0002 & -0.0001 & 0 & 0 \\
\hline U7 & 0 & 0 & 0 & 0 & 0 & 0 & 0 & 0 & 0.0037 & 0.0002 & 0.4761 & 0.0000 & 0.0037 & -0.0002 \\
\hline$\theta 7$ & 0 & 0 & 0 & 0 & 0 & 0 & 0 & 0 & -0.0002 & -0.0001 & 0.0000 & 0.0000 & 0.0002 & -0.0001 \\
\hline U8 & 0 & 0 & 0 & 0 & 0 & 0 & 0 & 0 & 0 & 0 & 0.0037 & 0.0002 & 4.66E-01 & -0.0004 \\
\hline$\theta 8$ & 0 & 0 & 0 & 0 & 0 & 0 & 0 & 0 & 0 & 0 & -0.0002 & -0.0001 & -0.0004 & 0.0000 \\
\hline
\end{tabular}

${ }^{1}$ Notice state sequence alternates translation, then rotation at each node

Table A4. Normalized mode shapes $\left[\times 10^{4}\right]$

\begin{tabular}{|c|c|c|c|c|c|c|c|c|c|c|c|c|c|}
\hline-1 & 3 & 2 & 0 & -3 & -5 & 3 & 1501 & 383 & 1037 & 443 & -692 & 181 & 240 \\
\hline-1097 & 3080 & 4481 & 4992 & 4505 & 3173 & -1154 & -4544 & -136 & 2388 & 2221 & 4049 & 1395 & 1669 \\
\hline-1 & 1 & -3 & -7 & -5 & 1 & -2 & -1569 & -215 & 204 & 667 & 1425 & -670 & -712 \\
\hline-2158 & 4857 & 3958 & 28 & -3814 & -4883 & 2208 & -943 & -2040 & -7064 & -867 & 912 & -2460 & -1864 \\
\hline-1 & -1 & -5 & 0 & 7 & 3 & 2 & 1296 & -125 & -1076 & 125 & 995 & -1385 & -1057 \\
\hline-3111 & 4495 & -1058 & -4992 & -1185 & 4481 & -3142 & 5806 & 2118 & 368 & -2572 & 4061 & -3204 & -683 \\
\hline 0 & 0 & 0 & 1 & 1 & -1 & 1 & -99 & 30 & 113 & -105 & 248 & 2247 & 954 \\
\hline-3902 & 2199 & -4861 & -47 & 4875 & -2071 & 3937 & -4288 & -3426 & 4504 & 1878 & 4753 & -3652 & 1689 \\
\hline 0 & -3 & 4 & 0 & -6 & 6 & 1 & 536 & -918 & 135 & 898 & 754 & -946 & 736 \\
\hline-4493 & -1062 & -3138 & 4985 & -3062 & -1232 & -4519 & 815 & 2292 & -2423 & 3091 & 891 & -3893 & 3998 \\
\hline 0 & 2 & 5 & -7 & 7 & -4 & 0 & -294 & 753 & 446 & 880 & 410 & -1936 & 1905 \\
\hline-4872 & -3903 & 2129 & 75 & -2232 & 3920 & 4832 & -2471 & 3385 & -210 & -3658 & 3392 & -4013 & 5178 \\
\hline-1 & -2 & 2 & -3 & 4 & -5 & -6 & 90 & -261 & 192 & -699 & -732 & -2945 & 3254 \\
\hline-5031 & -5052 & 5012 & -5030 & 5062 & -4984 & -4965 & 3580 & -7823 & 3941 & -7649 & -5153 & -4046 & 5502 \\
\hline
\end{tabular}

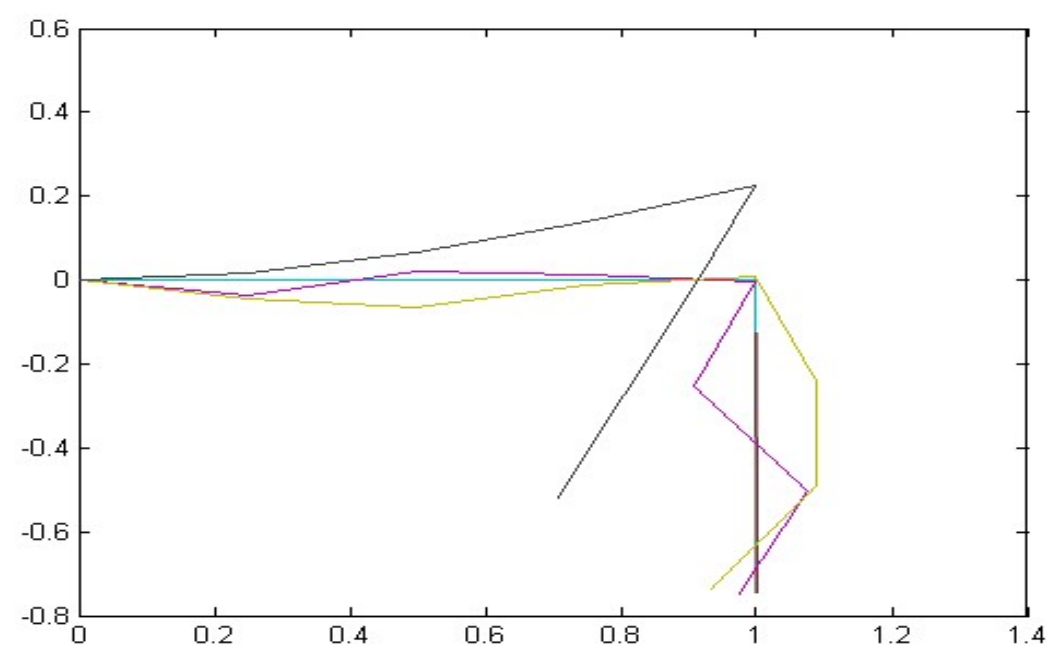

Figure A1. Normalized mode shapes (modal coordinates) displayed in physical coordinates with normalized length on the abscissa in meters and displacements in meters on the ordinant. 


\section{A.1 Equations of Motion in Standard State Space Form}

$$
\left\{\begin{array}{l}
\dot{x} \\
\ddot{x}
\end{array}\right\}_{[n \times 1]}=[A]_{n x n}\left\{\begin{array}{l}
x \\
\dot{x}
\end{array}\right\}_{n x 1}+[B]_{n x 1}\{u\}_{1 \times 1}
$$

1. Finite element analysis performed in MATLAB (code is included in the appendix) generates the mode shapes used to calculate the rigid-elastic coupling terms. The program outputs the flexible system [A], [B], [C], and [D] matrices of the standard state space form. The results are:

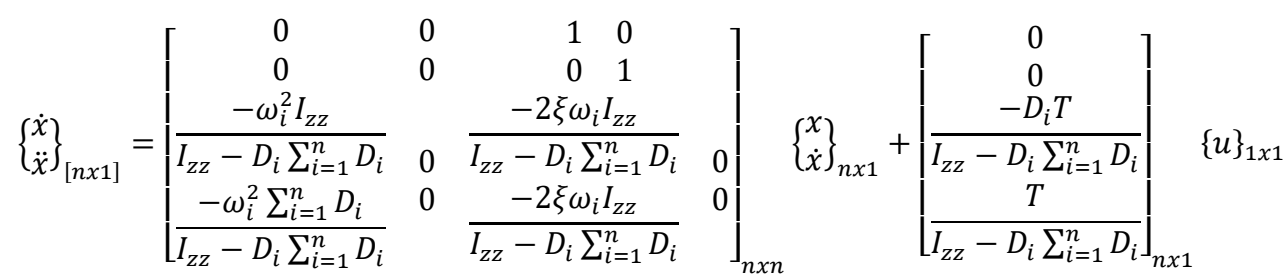

$$
\begin{aligned}
& {[C]=\left[\begin{array}{llllllllllll}
1 & 0 & 0 & 0 & 0 & 0 & 0 & 0 & 0 & 0 & 0 & 0
\end{array}\right] \quad[D]=[0]}
\end{aligned}
$$

Given these equations, the resultant state space matrices are:

Table A5. State Space [A] matrix

\begin{tabular}{c|cccccccccccc}
\hline & 1 & 2 & 3 & 4 & 5 & 6 & 7 & 8 & 9 & 10 & 11 \\
\hline 1 & 0 & 0 & 0 & 0 & 0 & 0 & 1 & 0 & 0 & 0 & 0 & 12 \\
2 & 0 & 0 & 0 & 0 & 0 & 0 & 0 & 1 & 0 & 0 & 0 & 0 \\
3 & 0 & 0 & 0 & 0 & 0 & 0 & 0 & 0 & 1 & 0 & 0 & 0 \\
4 & 0 & 0 & 0 & 0 & 0 & 0 & 0 & 0 & 0 & 1 & 0 & 0 \\
5 & 0 & 0 & 0 & 0 & 0 & 0 & 0 & 0 & 0 & 0 & 0 \\
6 & 0 & 0 & 0 & 0 & 0 & 0 & 0 & 0 & 0 & 0 & 0 & 1 \\
7 & 0 & 0.099 & -1.064 & -3.382 & -0.736 & -26.635 & 0 & $1.392 \mathrm{E}-04$ & $-5.066 \mathrm{E}-04$ & $-3.298 \mathrm{E}-04$ & $-4.662 \mathrm{E}-05$ & $-8.609 \mathrm{E}-04$ \\
8 & 0 & -0.659 & 1.642 & 5.218 & 1.136 & 41.100 & 0 & $-9.264 \mathrm{E}-04$ & $7.817 \mathrm{E}-04$ & $5.088 \mathrm{E}-04$ & $7.194 \mathrm{E}-05$ & $1.328 \mathrm{E}-03$ \\
9 & 0 & 0.188 & -6.435 & -6.433 & -1.401 & -50.670 & 0 & $2.649 \mathrm{E}-04$ & $-3.064 \mathrm{E}-03$ & $-6.273 \mathrm{E}-04$ & $-8.869 \mathrm{E}-05$ & $-1.638 \mathrm{E}-03$ \\
10 & 0 & 0.025 & -0.270 & -106.024 & -0.187 & -6.755 & 0 & $3.531 \mathrm{E}-05$ & $-1.285 \mathrm{E}-04$ & $-1.034 \mathrm{E}-02$ & $-1.182 \mathrm{E}-05$ & $-2.183 \mathrm{E}-04$ \\
11 & 0 & 0.002 & -0.025 & -0.079 & -249.447 & -0.620 & 0 & $3.241 \mathrm{E}-06$ & $-1.179 \mathrm{E}-05$ & $-7.677 \mathrm{E}-06$ & $-1.579 \mathrm{E}-02$ & $-2.004 \mathrm{E}-05$ \\
12 & 0 & 0.022 & -0.233 & -0.742 & -0.162 & -962.998 & 0 & $3.055 \mathrm{E}-05$ & $-1.112 \mathrm{E}-04$ & $-7.237 \mathrm{E}-05$ & $-1.023 \mathrm{E}-05$ & $-3.113 \mathrm{E}-02$ \\
\hline
\end{tabular}

${ }^{1}$ Flexible states where base (rigid body) rotation is controlled.

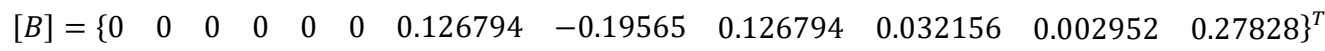

$$
\begin{aligned}
& {[C]=\left\{\begin{array}{llllllllllll}
1 & 0 & 0 & 0 & 0 & 0 & 0 & 0 & 0 & 0 & 0 & 0
\end{array}\right\} \quad[D]=[0]}
\end{aligned}
$$




\section{Appendix B Initialization Function callbacks for simulation depicted in figure 5}

This appendix is an optional section containing details supplemental to the main text crucial to understanding and reproducing the research.

clear all; close all; clc;

$\%$ This block of code establishes the properties of each beam element $\mathrm{a}=0.0254 ; \mathrm{b}=0.0016 ; \mathrm{L}=0.25$;

$\mathrm{E}=72^{*} 10^{\wedge} 9 ; \quad \mathrm{I}=\mathrm{a}^{*} \mathrm{~b}^{\wedge} 3 / 12 ;$

$\mathrm{Li}=\left[126^{*} \mathrm{~L}-126^{*} \mathrm{~L} ; 6^{*} \mathrm{~L} 4^{*} \mathrm{~L}^{\wedge} 2-6^{*} \mathrm{~L} 2^{*} \mathrm{~L}^{\wedge} 2 ;-12-6^{*} \mathrm{~L} 12-6^{*} \mathrm{~L} ; 6^{*} \mathrm{~L} 2^{*} \mathrm{~L}^{\wedge} 2-6^{*} \mathrm{~L} 4^{*} \mathrm{~L}^{\wedge} 2\right] ;$

k_beam $=\mathrm{E}^{*} \mathrm{I} / \mathrm{L}^{\wedge} 3^{*}[\mathrm{Li}]$;

rho_beam $=2.8^{*} 10^{\wedge} 3 ; \quad \%$ Beam density $\mathrm{kg} / \mathrm{m}^{\wedge} 3$

A_beam $=\mathrm{a} * \mathrm{~b}$; $\quad$ \%Beam cross sectional area

$\mathrm{mb}=$ rho_beam*A_beam; \%Beam mass per unit length

\%This block creates the empty stiffness matrix [k]

$\mathrm{k}=$ zeros $(14,14)$;

$\%$ This block fills in the stiffness matrix components

$\%$ Row 1 components start at index $=1$

$\mathrm{k}(1,1)=\mathrm{k} \_$beam $(3,3)+\mathrm{k} \_$beam $(1,1)$;

$\mathrm{k}(1,2)=\mathrm{k} \_$beam $(3,4)+\mathrm{k} \_$beam $(1,2)$;

$\mathrm{k}(1,3)=\mathrm{k} \_$beam $(1,3)$;

$\mathrm{k}(1,4)=\mathrm{k} \_$beam $(1,4)$;

$\%$ Row 3 components start at index $=29$

$\mathrm{k}(3,1)=\mathrm{k} \_$beam $(3,1)$;

$\mathrm{k}(3,2)=\mathrm{k}$ _beam $(3,2)$;

$\mathrm{k}(3,3)=\mathrm{k} \_$beam $(3,3)+\mathrm{k} \_$beam $(1,1)$;

$\mathrm{k}(3,4)=\mathrm{k}$ _beam $(3,4)+\mathrm{k}$ _beam $(1,2)$;

$\mathrm{k}(3,5)=\mathrm{k} \_$beam $(1,3)$;

$\mathrm{k}(3,6)=\mathrm{k} \_$beam $(1,4)$;

$\%$ Row 5 components start at index $=59$

$\mathrm{k}(5,3)=\mathrm{k} \_$beam $(3,1)$;

$\mathrm{k}(5,4)=\mathrm{k} \_$beam $(3,2)$;

$\mathrm{k}(5,5)=\mathrm{k} \_$beam $(3,3)+\mathrm{k} \_$beam $(1,1)$;

$\mathrm{k}(5,6)=\mathrm{k} \_$beam $(3,4)+\mathrm{k} \_$beam $(1,2)$;

$\mathrm{k}(5,7)=\mathrm{k} \_$beam $(1,3)$;

$\mathrm{k}(5,8)=\mathrm{k} \_$beam $(1,4)$;

$\%$ Row 7 components start at index $=89$

$\mathrm{k}(7,5)=\mathrm{k} \_$beam $(3,1)$;

$\mathrm{k}(7,6)=\mathrm{k} \_$beam $(3,2)$;

$\mathrm{k}(7,7)=\mathrm{k} \_$beam $(3,3)$;

$\mathrm{k}(7,8)=\mathrm{k} \_$beam $(3,4)$;
$\%$ Row 2 components start at index $=15$

$\mathrm{k}(2,1)=\mathrm{k} \_$beam $(4,3)+\mathrm{k} \_$beam $(2,1)$;

$\mathrm{k}(2,2)=\mathrm{k} \_$beam $(4,4)+\mathrm{k} \_$beam $(2,2)$;

$\mathrm{k}(2,3)=\mathrm{k}$ _beam $(2,3)$;

$\mathrm{k}(2,4)=\mathrm{k} \_$beam $(2,4)$;

$\%$ Row 4 components start at index $=43$

$\mathrm{k}(4,1)=\mathrm{k}$ _beam $(4,1)$;

$\mathrm{k}(4,2)=\mathrm{k}$ _beam $(4,2)$;

$\mathrm{k}(4,3)=\mathrm{k}$ bbeam $(4,3)+\mathrm{k} \_$beam $(2,1)$;

$\mathrm{k}(4,4)=\mathrm{k} \_$beam $(4,4)+\mathrm{k} \_$beam $(2,2)$;

$\mathrm{k}(4,5)=\mathrm{k}$ _beam $(2,3)$;

$\mathrm{k}(4,6)=\mathrm{k} \_$beam $(2,4)$;

\% Row 6 components start at index $=73$

$\mathrm{k}(6,3)=\mathrm{k} \_$beam $(4,1)$;

$\mathrm{k}(6,4)=\mathrm{k}$ _beam $(4,2)$;

$\mathrm{k}(6,5)=\mathrm{k} \_$beam $(4,3)+\mathrm{k} \_$beam $(2,1)$;

$\mathrm{k}(6,6)=\mathrm{k} \_$beam $(4,4)+\mathrm{k} \_$beam $(2,2)$;

$\mathrm{k}(6,7)=\mathrm{k}$ _beam $(2,3)$;

$\mathrm{k}(6,8)=\mathrm{k} \_$beam $(2,4)$;

$\%$ Row 8 components start at index $=103$

$\mathrm{k}(8,5)=\mathrm{k} \_$beam $(4,1)$;

$\mathrm{k}(8,6)=\mathrm{k}$ _beam $(4,2)$;

$\mathrm{k}(8,7)=\mathrm{k} \_$beam $(4,3)$;

$\mathrm{k}(8,8)=\mathrm{k} \_$beam $(4,4)+\mathrm{k} \_$beam $(2,2)$;

$\mathrm{k}(8,9)=\mathrm{k} \_$beam $(2,3)$;

$\mathrm{k}(8,10)=\mathrm{k} \_$beam $(2,4)$;

$\%$ Row 10 components start at index $=134$

$\mathrm{k}(10,8)=\mathrm{k} \_$beam $(4,2)$;

$\mathrm{k}(10,9)=\mathrm{k}$ _beam $(4,3)+\mathrm{k} \_$beam $(2,1)$;

$\mathrm{k}(10,10)=\mathrm{k} \_$beam $(4,4)+\mathrm{k} \_$beam $(2,2)$;
$\%$ Row 9 components start at index $=120$

$\mathrm{k}(9,8)=\mathrm{k} \_$beam $(3,2)$;

$\mathrm{k}(9,9)=\mathrm{k} \_$beam $(3,3)+\mathrm{k} \_$beam $(1,1)$;

$\mathrm{k}(9,10)=\mathrm{k} \_$beam $(3,4)+\mathrm{k} \_$beam $(1,2)$; 
$\mathrm{k}(9,11)=\mathrm{k} \_$beam $(1,3)$;

$\mathrm{k}(9,12)=\mathrm{k} \_$beam $(1,4)$;

$\%$ Row 11 components start at index $=149$

$\mathrm{k}(11,9)=\mathrm{k} \_$beam $(3,1)$;

$\mathrm{k}(11,10)=\mathrm{k} \_$beam $(3,2)$;

$\mathrm{k}(11,11)=\mathrm{k} \_$beam $(3,3)+\mathrm{k} \_$beam $(1,1)$;

$\mathrm{k}(11,12)=\mathrm{k} \_$beam $(3,4)+\mathrm{k} \_$beam $(1,2)$;

$\mathrm{k}(11,13)=\mathrm{k} \_$beam $(1,3)$;

$\mathrm{k}(11,14)=\mathrm{k} \_$beam $(1,4)$;

\% Row 13 components start at index=179

$\mathrm{k}(13,11)=\mathrm{k} \_$beam $(3,1)$;

$\mathrm{k}(13,12)=\mathrm{k}$ _beam $(3,2)$;

$\mathrm{k}(13,13)=\mathrm{k} \_$beam $(3,3)$;

$\mathrm{k}(13,14)=\mathrm{k} \_$beam $(3,4)$; $\mathrm{k}(10,11)=\mathrm{k} \_$beam $(2,3)$;

$\mathrm{k}(10,12)=\mathrm{k} \_$beam $(2,4)$;

\% Row 12 components start at index $=163$

$\mathrm{k}(12,9)=\mathrm{k} \_$beam $(4,1)$;

$\mathrm{k}(12,10)=\mathrm{k} \_$beam $(4,2)$;

$\mathrm{k}(12,11)=\mathrm{k} \_$beam $(4,3)+\mathrm{k} \_$beam $(2,1)$;

$\mathrm{k}(12,12)=\mathrm{k} \_$beam $(4,4)+\mathrm{k} \_$beam $(2,2)$;

$\mathrm{k}(12,13)=\mathrm{k} \_$beam $(2,3)$;

$\mathrm{k}(12,14)=\mathrm{k} \_$beam $(2,4)$;

$\%$ Row 14 components start at index $=193$

$\mathrm{k}(14,11)=\mathrm{k} \_$beam $(4,1)$;

$\mathrm{k}(14,12)=\mathrm{k} \_$beam $(4,2)$;

$\mathrm{k}(14,13)=\mathrm{k} \_$beam $(4,3)$;

$\mathrm{k}(14,14)=\mathrm{k} \_$beam $(4,4)$;

\%Display stiffness matrix to check

$\mathrm{k}=\mathrm{k}$;

\%END STIFFNESS MATRIX. START MASS MATRIX

\%Assemble individual beam inertia matrix

I_beam=ones $(1,8)$; $\quad$ \%Creates empty matrix of I's for eight node points

I_beam=[I_beam.*I]; \%Fill in matrix values with beam inertia

I_beam $(1)=0$;

$\%$ First node point inertia $=0$

\%This block of code creates the individual beam mass matrix "m_beam"

$\mathrm{mi}=\left[15622^{*} \mathrm{~L} 54-13^{*} \mathrm{~L} ; 22^{*} \mathrm{~L} 4^{*} \mathrm{~L}^{\wedge} 213^{*} \mathrm{~L}-3^{*} \mathrm{~L}^{\wedge} 2 ; 5413^{*} \mathrm{~L} 156-22^{*} \mathrm{~L} ;-13^{*} \mathrm{~L}-3^{*} \mathrm{~L}^{\wedge} 2-22^{*} \mathrm{~L} 4^{*} \mathrm{~L}^{\wedge} 2\right]$;

$\mathrm{m} \_$beam $=\mathrm{mb}^{*} \mathrm{~L} / 420^{*} \mathrm{mi}$;

\%This block of code establishes the value of each point mass (mp)

$\%$ and the system point mass matrix $(\mathrm{M})$

$\mathrm{mp}=0.455$; $\quad$ \%Point masses, $\mathrm{M}$

$\mathrm{M}=\left[0 \mathrm{mp} \mathrm{mp} \mathrm{mp} 2^{*} \mathrm{mp} \mathrm{mp} \mathrm{mp} \mathrm{mp]; \quad \% Matrix} \mathrm{of} 8\right.$ point masses (0 First point mass)

$\%$ Creates a 14x14 empty mass matrix [m]

$\mathrm{m}=$ zeros $(14,14)$;

\%Fill in the system mass matrix components

\% Row 1 components start at index $=1 \quad \%$ Row 2 components start at index $=15$

$\mathrm{m}(1,1)=\mathrm{m} \_$beam $(3,3)+\mathrm{m} \_$beam $(1,1)+\mathrm{M}(2) ; \mathrm{m}(2,1)=\mathrm{m} \_$beam $(4,3)+\mathrm{m} \_$beam $(2,1)$;

$\mathrm{m}(1,2)=\mathrm{m} \_$beam $(3,4)+\mathrm{m} \_$beam $(1,2) ; \quad \mathrm{m}(2,2)=\mathrm{m} \_$beam $(4,4)+\mathrm{m} \_$beam $(2,2)$;

$\mathrm{m}(1,3)=\mathrm{m} \_$beam $(1,3)$;

$\mathrm{m}(1,4)=\mathrm{m} \_$beam $(1,4)$;

$\mathrm{m}(2,3)=\mathrm{m} \_$beam $(2,3)$;

$\mathrm{m}(2,4)=\mathrm{m} \_$beam $(2,4)$;

$\%$ Row 3 components start at index $=29$

$\mathrm{m}(3,1)=\mathrm{m} \_$beam $(3,1)$;

$\%$ Row 4 components start at index $=43$

$\mathrm{m}(3,2)=\mathrm{m} \_$beam $(3,2)$;

$\mathrm{m}(4,1)=\mathrm{m} \_$beam $(4,1)$;

$\mathrm{m}(4,2)=\mathrm{m} \_$beam $(4,2)$;

$\mathrm{m}(3,3)=\mathrm{m}$ _beam $(3,3)+\mathrm{m} \_$beam $(1,1)+\mathrm{M}(3)$;

$\mathrm{m}(3,4)=\mathrm{m} \_$beam $(3,4)+\mathrm{m} \_$beam $(1,2)$;

$\mathrm{m}(3,5)=\mathrm{m} \_$beam $(1,3)$;

$\mathrm{m}(3,6)=\mathrm{m} \_$beam $(1,4)$;

$\mathrm{m}(4,3)=m \_$beam $(4,3)+\mathrm{m} \_$beam $(2,1)$;

$\mathrm{m}(4,4)=\mathrm{m} \_$beam $(4,4)+\mathrm{m} \_$beam $(2,2)$;

$\mathrm{m}(4,5)=\mathrm{m} \_$beam $(2,3)$;

$\mathrm{m}(4,6)=\mathrm{m} \_$beam $(2,4)$; 
\% Row 5 components start at index=59

$\mathrm{m}(5,3)=\mathrm{m} \_$beam $(3,1)$;

$\mathrm{m}(5,4)=\mathrm{m} \_$beam $(3,2)$;

$\mathrm{m}(5,5)=\mathrm{m} \_$beam $(3,3)+\mathrm{m} \_$beam $(1,1)+\mathrm{M}(4)$;

$\mathrm{m}(5,6)=\mathrm{m} \_$beam $(3,4)+\mathrm{m} \_$beam $(1,2)$;

$\mathrm{m}(5,7)=\mathrm{m} \_$beam $(1,3)$;

$\mathrm{m}(5,8)=\mathrm{m} \_$beam $(1,4)$;
\% Row 6 components start at index $=73$

$\mathrm{m}(6,3)=\mathrm{m} \_$beam $(4,1)$;

$\mathrm{m}(6,4)=\mathrm{m} \_$beam $(4,2)$;

$\mathrm{m}(6,5)=\mathrm{m} \_$beam $(4,3)+\mathrm{m} \_$beam $(2,1)$;

$\mathrm{m}(6,6)=\mathrm{m} \_$beam $(4,4)+\mathrm{m} \_$beam $(2,2)$;

$\mathrm{m}(6,7)=\mathrm{m} \_$beam $(2,3)$;

$\mathrm{m}(6,8)=\mathrm{m} \_$beam $(2,4)$;

\% Row 7 components start at index $=89$

$\mathrm{m}(7,5)=\mathrm{m} \_$beam $(3,1)$;

$\mathrm{m}(7,6)=\mathrm{m} \_$beam $(3,2)$;

$\mathrm{m}(7,7)=\mathrm{m} \_$beam $(3,3)+3^{*} \mathrm{mb}+\mathrm{M}(5)+\mathrm{M}(6)+\mathrm{M}(7)+\mathrm{M}(8)$;

$\mathrm{m}(7,8)=\mathrm{m} \_$beam $(3,4)$;

\% Row 8 components start at index $=103$

$\mathrm{m}(8,5)=\mathrm{m} \_$beam $(4,1)$;

$\mathrm{m}(8,6)=\mathrm{m} \_$beam $(4,2)$;

$\mathrm{m}(8,7)=\mathrm{m} \_$beam $(4,3)$;

$\mathrm{m}(8,8)=\mathrm{m} \_$beam $(4,4)+\mathrm{m} \_$beam $(2,2)$;

$\mathrm{m}(8,9)=\mathrm{m} \_$beam $(2,3)$;

$\mathrm{m}(8,10)=\mathrm{m} \_$beam $(2,4)$;

\% Row 10 components start at index $=134$

$\mathrm{m}(10,8)=\mathrm{m} \_$beam $(4,2)$;

$\mathrm{m}(10,9)=$ m_beam $(4,3)+\mathrm{m} \_$beam $(2,1)$;

$\mathrm{m}(10,10)=\mathrm{m} \_$beam $(4,4)+\mathrm{m} \_$beam $(2,2)$;

$\mathrm{m}(11,11)=\mathrm{m} \_$beam $(3,3)+\mathrm{m} \_$beam $(1,1)+\mathrm{M}(7)$;

$\mathrm{m}(10,11)=\mathrm{m} \_$beam $(2,3)$;

$\mathrm{m}(10,12)=\mathrm{m} \_$beam $(2,4)$;

\% Row 9 components start at index $=120$

$\mathrm{m}(9,8)=\mathrm{m} \_$beam $(3,2)$;

$\mathrm{m}(9,9)=\mathrm{m} \_$beam $(3,3)+\mathrm{m} \_$beam $(1,1)+\mathrm{M}(6)$;

$\mathrm{m}(9,10)=\mathrm{m} \_$beam $(3,4)+\mathrm{m} \_$beam $(1,2)$;

$\mathrm{m}(9,11)=\mathrm{m} \_$beam $(1,3)$;

$\mathrm{m}(9,12)=\mathrm{m} \_$beam $(1,4)$;

\% Row 11 components start at index $=149$

$\mathrm{m}(11,9)=\mathrm{m} \_$beam $(3,1)$;

$\mathrm{m}(11,10)=\mathrm{m} \_$beam $(3,2)$;

$\mathrm{m}(11,12)=\mathrm{m} \_$beam $(3,4)+\mathrm{m} \_$beam $(1,2)$;

$\mathrm{m}(11,13)=\mathrm{m} \_$beam $(1,3)$;

$\mathrm{m}(11,14)=\mathrm{m} \_$beam $(1,4)$;

$\%$ Row 12 components start at index $=163$

$\mathrm{m}(12,9)=\mathrm{m} \_$beam $(4,1)$;

$\mathrm{m}(12,10)=\mathrm{m} \_$beam $(4,2)$;

$\mathrm{m}(12,11)=\mathrm{m} \_$beam $(4,3)+\mathrm{m} \_$beam $(2,1)$;

$\mathrm{m}(12,12)=\mathrm{m} \_$beam $(4,4)+\mathrm{m} \_$beam $(2,2)$;

$\mathrm{m}(12,13)=\mathrm{m} \_$beam $(2,3)$;

$\mathrm{m}(12,14)=\mathrm{m} \_$beam $(2,4)$;

\% Row 13 components start at index $=179$

$\mathrm{m}(13,11)=\mathrm{m} \_$beam $(3,1)$;

\% Row 14 components start at index=193

$\mathrm{m}(13,12)=\mathrm{m} \_$beam $(3,2)$;

$\mathrm{m}(14,11)=\mathrm{m} \_$beam $(4,1)$;

$\mathrm{m}(13,13)=\mathrm{m} \_$beam $(3,3)+\mathrm{M}(8)$;

$\mathrm{m}(14,12)=\mathrm{m} \_$beam $(4,2)$;

$\mathrm{m}(14,13)=\mathrm{m} \_$beam $(4,3)$;

$\mathrm{m}(13,14)=\mathrm{m} \_$beam $(3,4)$;

$\mathrm{m}(14,14)=\mathrm{m} \_$beam $(4,4)$;

\%Display the system mass matrix to check $\mathrm{m}=\mathrm{m}$;

$\%$ Calculate the natural frequencies and normal modes

[NormalModes, EigenValues] =eig $\left(\operatorname{inv}(\mathrm{m})^{*} \mathrm{k}\right)$;

NaturalFrequencies $=\operatorname{diag}\left(\right.$ EigenValues $\left.^{\wedge} 0.5\right)$;

ModeShapes=NormalModes;

\%Check Orthogonality like Homework 1 confirm diagonal matrix of 1 's

\%to satisfy equation 24 on slide 17 
OrthoMass=NormalModes'* ${ }^{*}$ NormalModes;

OrthoStiff $=$ NormalModes' ${ }^{*}{ }^{*}$ NormalModes;

StiffCheck=OrthoStiff/EigenValues;

Equation24_OrthoCheck=diag(diag(StiffCheck/OrthoMass));

\%Spacecraft Radius to be used designating rigid modal coordinate

$\mathrm{R}=0.381$;

FeeE=NormalModes; $\quad$ \%Designate Elastic mode shapes array FeeE

Omega=NaturalFrequencies; \%Designate variable name 'Omega' as natural frequencies

$\%$ Designate Rigid modal coordinate FeeR

FeeR=[R+L 1 R+L*2 1 R+L*3 1 R+L*4 1 -L 1 -L*2 1 -L*3 1];

$\mathrm{Di}=\mathrm{FeeE}^{* *} \mathrm{~m}^{*} \operatorname{diag}(\mathrm{FeeR}) ; \quad \%$ Calculate Rigid-Elastic Coupling Coefficient

$\operatorname{DiCheck}=\operatorname{det}(\mathrm{Di})$;

$\%$ Confirm Di is singular...det(Di=0)

$\mathrm{Z}=0.0005$;

$\mathrm{Izz}=14$;

$\mathrm{w}=\operatorname{diag}($ NaturalFrequencies); $\%$ Generate a diagonal matrix of natural frequency

$\mathrm{Iw}=0.0912$;

$\mathrm{Td}=0$;

$\mathrm{Tc}=0.1$;

$\%$ Disturbance Torque

$\mathrm{T}=\mathrm{Td}+\mathrm{Tc}$;

$\%$ Control Torque is Iw* ${ }^{*}$ dddot_wheel

$\%$ Total Torque is sum of disturbance and control torques

\%Start State Space Development

NatFreq $=\operatorname{diag}($ EigenValues $) .^{\wedge} 0.5$;

$\mathrm{r}=0.381$;

$\%$ Radius of the wheel (large rigid body)

freqs $=$ sqrt(EigenValues); \%

NatFreq = EigenValues $(1: 5,1: 5)$;

freqs $=$ freqs $(1: 5,1: 5)$;

zeta $=0.0005$;

\%Given damping ratio for all modes

$\mathrm{Izz}=14$;

phi_E $=$ NormalModes(1:14,1:5);

phi_R $=\left[\mathrm{r}+\mathrm{L}, 1, \mathrm{r}+2^{*} \mathrm{~L}, 1, \mathrm{r}+3^{*} \mathrm{~L}, 1, \mathrm{r}+4^{*} \mathrm{~L}, 1,-\mathrm{L}, 1,-2^{*} \mathrm{~L}, 1,-3^{*} \mathrm{~L}, 1\right]^{\prime}$;

M_II = m;

$\mathrm{Di}=[$ phi_E'* M_II*phi_R];

M_state $=[$ Izz Di';

Di eye(5)];

C_damp $=[$ zeros $(6,6)]$;

C_damp $(2: 6,2: 6)=2^{*}$ zeta $^{*}$ freqs;

$\mathrm{K}=[\operatorname{zeros}(6,6)]$;

$\mathrm{K}(2: 6,2: 6)=$ NatFreq;

$A=[\operatorname{zeros}(6)$, eye $(6,6)$;

-inv(M_state $)^{*} \mathrm{~K},-\operatorname{inv}\left(\mathrm{M} \_ \text {state }\right)^{*} \mathrm{C} \_$damp];

Bprime $=[1 ; 0 ; 0 ; 0 ; 0 ; 0]$;

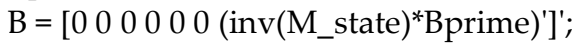

$\mathrm{C}=\operatorname{zeros}(12,12) ; \mathrm{C}(1,1)=1$;

$\mathrm{D}=\operatorname{zeros}(12,1)$;

[Gnum,Gden] = ss2tf $(\mathrm{A}, \mathrm{B}, \mathrm{C}, \mathrm{D})$;

$\mathrm{G} 1=\operatorname{tf}(\operatorname{Gnum}(1,:), \mathrm{Gden})$

\%Manually input Transfer Function to check 
NUM=[1.998e-015 0.1268 0.007582 166.9 5.591 4.718e004 771 3.412e006 1.218e004 1.576e007 $1.475 \mathrm{e} 004$ 7.11e006];

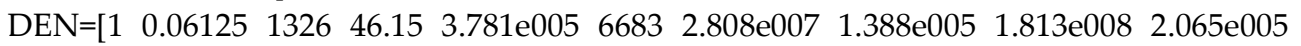
$9.954 \mathrm{e} 00700]$;

$\mathrm{G}=\mathrm{tf}(\mathrm{NUM}, \mathrm{DEN})$;

\%Put PID controller Transfer function into workspace

$\mathrm{It}=14 ; \mathrm{Z}=0.516931$;

Bandwidth=4; wn=Bandwidth; $\mathrm{T}=10 / \mathrm{Z} / \mathrm{wn}$;

$\mathrm{Kd}=2^{*} \mathrm{Z}^{*} \mathrm{wn}{ }^{*} \mathrm{It}+\mathrm{It} / \mathrm{T}$;

$\mathrm{Kp}=\mathrm{wn}^{\wedge} 2+2^{*} \mathrm{Z}^{*} \mathrm{wn} / \mathrm{T}$;

$\mathrm{Ki}=\mathrm{wn}^{\wedge} 2 / \mathrm{T}$;

$\mathrm{PID}=\mathrm{tf}\left([\mathrm{Kd} \mathrm{Kp} \mathrm{Ki}],\left[\begin{array}{lll}0 & 1 & 0\end{array}\right]\right)$;

\%DESIGN FILTERS TO SMOOTH OUT MODE 1

$\%$ Design Bandpass filter for $\mathrm{w}=10^{\wedge}-0.1478=0.711541 \mathrm{~Hz}$

$\mathrm{wZ}=0.711541 ; \mathrm{Zz}=0.1 ; \mathrm{wp}=\mathrm{wz} ; \mathrm{Zp}=0.0005$;

$\mathrm{BP} 1=\mathrm{tf}\left(\left[1 / \mathrm{wz}^{\wedge} 22^{*} \mathrm{Zz} / \mathrm{wz} 1\right],\left[1 / \mathrm{wp}^{\wedge} 22^{*} \mathrm{Zp} / \mathrm{wp} 1\right]\right)$;

PID_BP1=PID*BP1;

$\%$ Design Notch filter for $w=10^{\wedge}-0.109=0.778037 \mathrm{~Hz}$

$\mathrm{wz}=0.778037 ; \mathrm{Zz}=0.0005 ; \mathrm{wp}=\mathrm{wz} ; \mathrm{Zp}=0.1$;

Notch1 $=\mathrm{tf}\left(\left[1 / \mathrm{wz}^{\wedge} 22^{*} \mathrm{Zz} / \mathrm{wz} 1\right],\left[1 / \mathrm{wp}^{\wedge} 22^{*} \mathrm{Zp} / \mathrm{wp} 1\right]\right)$;

Mode_1=PID*BP1*Notch1;

\%DESIGN FILTERS TO SMOOTH OUT MODE 2

$\%$ Design Bandpass filter for $\mathrm{w}=10^{\wedge} 0.3223$

$\mathrm{wz}=10^{\wedge} 0.3223 ; \mathrm{Zz}=0.1 ; \mathrm{wp}=\mathrm{wz} ; \mathrm{Zp}=0.0005$;

$\mathrm{BP} 2=\mathrm{tf}\left(\left[1 / \mathrm{wz}^{\wedge} 22^{*} \mathrm{Zz} / \mathrm{wz} 1\right],\left[1 / \mathrm{wp}^{\wedge} 22^{*} \mathrm{Zp} / \mathrm{wp} 1\right]\right)$;

$\%$ Design Notch filter for $\mathrm{w}=10^{\wedge} 0.405$

$\mathrm{wz}=10^{\wedge} 0.405 ; \mathrm{Zz}=0.0006 ; \mathrm{wp}=\mathrm{wz} ; \mathrm{Zp}=0.1$;

Notch2=tf([1/wz^2 2*Zz/wz 1],[1/wp $\left.\left.{ }^{\wedge} 22^{*} \mathrm{Zp} / \mathrm{wp} 1\right]\right)$;

Mode_2=Mode_1*BP2*Notch2;

$\%$ Design Lead filter for $w z \sim 1, w p \sim 3$

$\% \mathrm{wz}=1 ; \mathrm{Zz}=1 ; \mathrm{wp}=3 ; \mathrm{Zp}=1$;

$\%$ Lead $=\operatorname{tf}\left(\left[1 / w^{\wedge} 22^{*} Z z / w z ~ 1\right],\left[1 / w^{\wedge} 22^{*} Z p / w p ~ 1\right]\right) ;$

$\%$ Mode_2=Mode_2*Lead;

\%DESIGN FILTERS TO SMOOTH OUT MODE 3

$\%$ Design Bandpass filter for $\mathrm{w}=10^{\wedge} 1.0110$

$\mathrm{wZ}=10^{\wedge} 1.0110 ; \mathrm{Zz}=0.1 ; \mathrm{wp}=\mathrm{wz} ; \mathrm{Zp}=0.0005$;

$\mathrm{BP} 3=\mathrm{tf}\left(\left[1 / \mathrm{wz}^{\wedge} 22^{*} \mathrm{Zz} / \mathrm{wz} 1\right],\left[1 / \mathrm{wp}^{\wedge} 22^{*} \mathrm{Zp} / \mathrm{wp} 1\right]\right)$;

$\%$ Design Notch filter for $\mathrm{w}=10^{\wedge} 1.0128$

$\mathrm{wz}=10^{\wedge} 1.0128 ; \mathrm{Zz}=0.0005 ; \mathrm{wp}=\mathrm{wz} ; \mathrm{Zp}=0.1$;

Notch3 $=\mathrm{tf}\left(\left[1 / \mathrm{wz}^{\wedge} 22^{*} \mathrm{Zz} / \mathrm{wz} 1\right],\left[1 / \mathrm{wp}^{\wedge} 22^{*} \mathrm{Zp} / \mathrm{wp} 1\right]\right)$;

Mode_3=Mode_2*BP3*Notch3;

\%DESIGN FILTERS TO SMOOTH OUT MODE 4

$\%$ Design Bandpass filter for $\mathrm{w}=10^{\wedge} 1.49035$

$\mathrm{wZ}=10^{\wedge} 1.49035 ; \mathrm{Zz}=0.1 ; \mathrm{wp}=\mathrm{wz} ; \mathrm{Zp}=0.0005$;

$\mathrm{BP} 4=\mathrm{tf}\left(\left[1 / \mathrm{wz}^{\wedge} 22^{*} \mathrm{Zz} / \mathrm{wz} 1\right],\left[1 / \mathrm{wp}^{\wedge} 22^{*} \mathrm{Zp} / \mathrm{wp} 1\right]\right)$; 
$\%$ Design Notch filter for $\mathrm{w}=10^{\wedge} 1.492$

$\mathrm{wz}=10^{\wedge} 1.492 ; \mathrm{Zz}=0.0005 ; \mathrm{wp}=\mathrm{wz} ; \mathrm{Zp}=0.1$;

Notch4=tf([1/wz^2 2*Zz/wz 1],[1/wp^ $\left.\left.22^{*} Z \mathrm{Zp} / \mathrm{wp} 1\right]\right) ;$

Mode_4=Mode_3*BP4*Notch4;

\%CALCULATE SYSTEM NATURAL FREQUENCIES

[NaturalFrequencies,Damping,EigenValue] $=\mathrm{damp}(\mathrm{G})$;

NaturalFrequencies=NaturalFrequencies;

\section{Appendix C Stop Function callbacks for simulation in figure 5}

This appendix is an optional section containing details supplemental to the main text crucial to understanding and reproducing the research.

[mag1,phase1,wout1] = bode(G); Mag1=20*log10(mag1(:)); Phase1=phase1(:);

[mag2, phase2,wout2] = bode(G*PID); Mag2=20*log10(mag2(:)); Phase2=phase2(:);

[mag3, phase3, wout3] = bode(G*PID*BP1); Mag3=20*log10(mag3(:)); Phase3=phase3(:);

[mag4, phase4,wout4] = bode(G*PID*BP1*Notch1); Mag4=20*log10(mag4(:)); Phase4=phase4(:);

[mag5,phase5,wout5] = bode(G*PID*BP1*Notch1*BP2); $\quad$ Mag5=20*log10(mag5(:));

Phase5=phase5(:);

$[$ mag6, phase6, wout6 $]=\operatorname{bode}\left(\mathrm{G}^{*} \mathrm{PID}^{*} \mathrm{BP} 1^{*}\right.$ Notch1*BP2*Notch2 $) ; \quad$ Mag6=20*log10 $(\operatorname{mag} 6(:))$;

Phase6=phase6(:);

$\left[\right.$ mag7,phase7,wout7] = bode $\left(G^{*}\right.$ PID $^{*}$ BP1*Notch1*BP2*Notch2*BP3); $\quad$ Mag7=20*log10(mag7(:)); Phase7=phase7(:);

[mag8,phase8,wout8]

$=$

bode(G*PID*BP1*Notch1*BP2*Notch2*BP3*Notch3);

Mag8=20*log10(mag8(:)); Phase8=phase8(:);

[mag9,phase9, wout9] = $\quad$ bode $\left(\mathrm{G}^{*} \mathrm{PID}^{*}{ }^{\mathrm{BP}} 1^{*}\right.$ Notch1*BP2*Notch2*BP3*Notch3*BP4); Mag9=20*log10(mag9(:)); Phase9=phase9(:);

[mag10,phase10, wout10] = bode $\left(\mathrm{G}^{*} \mathrm{PID}^{*} \mathrm{BP} 1{ }^{*}\right.$ Notch1*BP2*Notch2*BP3*Notch3*BP4*Notch4); Mag10=20* $\log 10(\operatorname{mag} 10(:)) ;$ Phase10=phase10(:);

figure(1); hold on;

semilogx(wout1,Mag1,'--','LineWidth',1);

semilogx(wout2,Mag2,'LineWidth',1);

semilogx(wout3,Mag3,'--','LineWidth',3);

semilogx(wout4,Mag4,':','LineWidth',2);

hold off; grid on; axis([0.5,40, -100, 150 ]); set(gca, 'FontSize',28, 'FontName','Palatino Linotype');

legend('Flexible space robot','PID','PID + Bandpass','PID + Notch + Bandpass')

figure(2); hold on; set(gca, 'FontSize',28, 'FontName','Palatino Linotype');

semilogx(wout1,Phase1,'--','LineWidth',1);

semilogx(wout2,Phase2,'LineWidth',1);

semilogx(wout3,Phase3,'--','LineWidth',3);

semilogx(wout4,Phase4,':','LineWidth',2);

hold off; grid on;

figure(3); hold on;

semilogx(wout2,Mag2,'--','LineWidth',1);

semilogx(wout4,Mag4,'LineWidth',1);

semilogx(wout5,Mag5,'--','LineWidth',3);

semilogx(wout6,Mag6,':','LineWidth',2);

hold off; grid on; axis([0.5,40, -100, 150 ]); set(gca, 'FontSize',28, 'FontName','Palatino Linotype');

legend('PID controlled Flexible space robot','PID+Mode 1','PID + Mode 1 + Bandpass','PID + Mode $1+$ Notch + Bandpass') 
figure(4); hold on; set(gca, 'FontSize',28, 'FontName','Palatino Linotype'); semilogx(wout2,Phase2,'--','LineWidth',1); semilogx(wout4,Phase4,'LineWidth',1); semilogx(wout5,Phase5,'--','LineWidth',3); semilogx(wout6,Phase6,':','LineWidth',2); hold off; grid on;

figure(5); hold on; semilogx(wout2,Mag2,'--','LineWidth',1); semilogx(wout4,Mag4,'LineWidth',1); semilogx(wout6,Mag6,'--','LineWidth',3); semilogx(wout7,Mag7,':','LineWidth',2); semilogx(wout8,Mag8,':','LineWidth',2); hold off; grid on; axis([0.5,40, -100, 150 ]); set(gca, 'FontSize',28, 'FontName','Palatino Linotype'); legend('PID controlled Flexible space robot', 'PID + Mode 1','PID + Mode 2','PID + Mode 1 + Mode 2 + Bandpass','PID + Mode $1+$ Mode 2 + Bandpass + Notch')

figure(6); hold on; set(gca, 'FontSize',28, 'FontName','Palatino Linotype'); semilogx(wout2,Phase2,'--','LineWidth',1); semilogx(wout4,Phase4,'LineWidth',1); semilogx(wout6,Phase6,'--','LineWidth',3); semilogx(wout7,Phase7,':','LineWidth',2); semilogx(wout8,Phase8, ':','LineWidth',2); hold off; grid on;

figure(7); hold on; semilogx(wout2,Mag2,'--','LineWidth',1); semilogx(wout4,Mag4,'LineWidth',1); semilogx(wout6,Mag6,'--','LineWidth',3); semilogx(wout8,Mag8,':','LineWidth',2); semilogx(wout9,Mag9,':','LineWidth',2); semilogx(wout10,Mag10,':','LineWidth',2); hold off; grid on; axis([0.5,40, -100, 150 ]); set(gca, 'FontSize',28, 'FontName','Palatino Linotype'); legend('PID controlled Flexible space robot', 'PID + Mode 1','PID + Mode 2','PID + Mode 1 + Mode 2 + Mode 3','PID + Mode $1+$ Mode 2 + Mode 3 + Bandpass + Notch')

figure(8); hold on; set(gca, 'FontSize',28, 'FontName','Palatino Linotype');

semilogx(wout2,Phase2,'--','LineWidth',1);

semilogx(wout4,Phase4,'LineWidth',1);

semilogx(wout6,Phase6,'--','LineWidth',3);

semilogx(wout8,Phase8,':','LineWidth',2);

semilogx(wout9,Phase9, ',','LineWidth',2);

semilogx(wout10,Phase10,':','LineWidth',2);

hold off; grid on;

sys1 $=G^{*} P I D /\left(1+G^{*} P I D\right)$;

sys $2=\left(G^{*} P I D * B P 1 /\left(1+G^{*} P I D * B P 1\right)\right)$;

sys $3=\left(G^{*} P I D * B P 1 *\right.$ Notch $1 /\left(1+G^{*} P I D * B P 1 *\right.$ Notch 1$\left.)\right)$;

sys $4=\left(\mathrm{G}^{*} \mathrm{PID}{ }^{*} \mathrm{BP} 1^{*}\right.$ Notch ${ }^{*} \mathrm{BP} 2 /\left(1+\mathrm{G}^{*} \mathrm{PID}{ }^{*} \mathrm{BP} 1{ }^{*}\right.$ Notch $\left.\left.1{ }^{*} \mathrm{BP} 2\right)\right)$;

sys5 $=\left(G^{*} P I D * B P 1 *\right.$ Notch $1 * B P 2 *$ Notch2 $/\left(1+G^{*} P I D * B P 1 *\right.$ Notch $1 * B P 2 *$ Notch 2$\left.)\right)$;

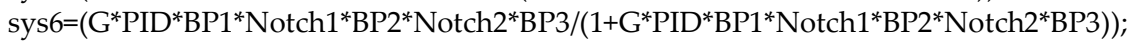

sys $7=\left(G^{*} P I D * B P 1{ }^{*}\right.$ Notch ${ }^{*} B P 2{ }^{*}$ Notch2 ${ }^{*} B P 3^{*}$ Notch $3 /\left(1+G^{*} P I D * B P 1{ }^{*}\right.$ Notch $1{ }^{*} B P 2{ }^{*}$ Notch2 ${ }^{*} B P{ }^{*}$ Notc h3));

sys $8=\left(G^{*}\right.$ PID*BP1*Notch1*BP2*Notch2*BP3*Notch3*BP4/(1+G*PID*BP1*Notch1*BP2*Notch2*BP3* Notch3*BP4));

sys9 $=\left(G^{*}\right.$ PID ${ }^{*} B P 1{ }^{*}$ Notch1*BP2*Notch2*BP3*Notch3*BP4*Notch4/(1+G*PID*BP1*Notch1*BP2*Notc h2*BP3*Notch3*BP4*Notch4));

figure(9); step(sys1,sys2); legend('PID','PID + BP1');set(gca, 'FontSize',28, 'FontName','Palatino Linotype'); 
figure(10); step(sys1,sys3); legend('PID','PID + BP1+Notch1'); set(gca, 'FontSize',28, 'FontName','Palatino Linotype');

figure(11); step(sys1,sys4); legend('PID','PID + Mode 1 + BP2'); set(gca, 'FontSize',28, 'FontName','Palatino Linotype');

figure(12); step(sys1,sys5); legend('PID','PID + Mode 1 + BP2 + Notch 2'); set(gca, 'FontSize',28, 'FontName','Palatino Linotype');

figure(13); step(sys1,sys6); legend('PID','PID + Mode 1 + Mode 2 + BP3'); set(gca, 'FontSize',28, 'FontName','Palatino Linotype');

figure(14); step(sys1,sys7); legend('PID','PID + Mode 1 + Mode 2 + BP3 + Notch3'); set(gca, 'FontSize',28, 'FontName','Palatino Linotype');

figure(15); step(sys1, sys8); legend('PID','PID + Mode 1 + Mode 2 + Mode 3 + BP4'); set(gca, 'FontSize',28, 'FontName','Palatino Linotype');

figure(16); step(sys1,sys9); legend('PID','PID + Mode 1 + Mode 2 + Mode 3 + BP4 + Notch4'); set(gca, 'FontSize',28, 'FontName','Palatino Linotype'); 